\title{
Piecewise Linear Systems with Closed Sliding Poly-Trajectories
}

\author{
Jaime R. de Moraes Paulo R. da Silva*
}

\begin{abstract}
In this paper we study piecewise linear (PWL) vector fields

$$
F(x, y)=\left\{\begin{array}{lll}
F^{+}(x, y) & \text { if } \quad x \geq 0 \\
F^{-}(x, y) & \text { if } \quad x \leq 0
\end{array}\right.
$$

where $\mathrm{x}=(x, y) \in \mathbb{R}^{2}, F^{+}(\mathrm{x})=A^{+} \mathrm{x}+b^{+}$and $F^{-}(\mathrm{x})=A^{-} \mathrm{x}+b^{-}, A^{+}=$ $\left(a_{i j}^{+}\right)$and $A^{-}=\left(a_{i j}^{-}\right)$are $(2 \times 2)$ constant matrices, $b^{+}=\left(b_{1}^{+}, b_{2}^{+}\right) \in \mathbb{R}^{2}$ and $b^{-}=\left(b_{1}^{-}, b_{2}^{-}\right) \in \mathbb{R}^{2}$ are constant vectors in $\mathbb{R}^{2}$. We suppose that the equilibrium points are saddle or focus in each half-plane. We establish a correspondence between the PWL vector fields and vectors formed by some of the following parameters: sets on $\Sigma$ (crossing, sliding or escaping), kind of equilibrium (real or virtual), intersection of manifolds with $\Sigma$, stability and orientation of the focus. Such vectors are called configurations. We reduce the number of configurations by an equivalent relation. Besides, we analyze for which configurations the corresponding PWL vector fields can have or not closed sliding poly-trajectories.
\end{abstract}

\section{Introduction}

There exist a lot of papers contributing to study of minimal sets of vector fields, such as, $[6,10]$. In this context, piecewise linear (PWL) vector fields are studied

*The first author is supported by FAPESP (number process: 2010/17956-1). The second author is partially supported by CAPES, CNPq-Brazil and FAPESP

Received by the editors in November 2013 - In revised form in January 2014.

Communicated by P. De Maesschalck.

2010 Mathematics Subject Classification : 37C10,34H99.

Key words and phrases : Piecewise linear systems, vector fields, poly-trajectories. 
by very researchers and serve as models for a great variety of engineering devices and biology, see for instance [8]. The simplest case is when there are two linearity regions delimited by a straight line. One of the pioneers in the study of (PWL) systems was Filippov that established conventions about the vector field in the region of discontinuity. After Filippov, others researches such as A. Gasull, S. Huan, X. Yang, J. Llibre, E. Ponce, among others, worked in this context.

Linear vector fields do not have limit cycles, but there are many examples of PWL vector fields with closed poly-trajectories (see Examples 3, 4 and 5). One of the main problems in the qualitative theory of planar differential equations is to identify the existence of the limit cycles and its number. This problem has been studied recently in PWL, for instance, in [2, 9].

Denote $\Sigma=\left\{(0, y) \in \mathbb{R}^{2}\right\}, \Sigma^{+}=\left\{(x, y) \in \mathbb{R}^{2}: x>0\right\}$ and $\Sigma^{-}=\{(x, y) \in$ $\left.\mathbb{R}^{2}: x<0\right\}$. Let $\Omega^{L}$ be the space of vector fields $F$ on $\mathbb{R}^{2}$ defined by:

$$
F(x, y)= \begin{cases}F^{+}(x, y) & \text { if } \quad x \geq 0 \\ F^{-}(x, y) & \text { if } \quad x \leq 0\end{cases}
$$

where, $F^{+}(\mathrm{x})=\left(F_{1}^{+}(\mathrm{x}), F_{2}^{+}(\mathrm{x})\right)=A^{+} \mathrm{x}+b^{+}$and $F^{-}(\mathrm{x})=\left(F_{1}^{-}(\mathrm{x}), F_{2}^{-}(\mathrm{x})\right)=$ $A^{-} \mathrm{x}+b^{-}, A^{+}=\left(a_{i j}^{+}\right)$and $A^{-}=\left(a_{i j}^{-}\right)$are $(2 \times 2)$ constant matrices and $b^{+}=$ $\left(b_{1}^{+}, b_{2}^{+}\right) \in \mathbb{R}^{2}$ and $b^{-}=\left(b_{1}^{-}, b_{2}^{-}\right) \in \mathbb{R}^{2}$ are constant vectors in $\mathbb{R}^{2}$.

We say that (1) is a piecewise linear (PWL) vector field and we denote $F \in \Omega^{L}$. We write $F=\left(F^{+}, F^{-}\right)$, which we will accept to be bi-valued in the points of $\Sigma$. Following Filippov's terminology, we distinguish the following sets on $\Sigma$ :

$$
\begin{aligned}
& \Sigma^{c}=\left\{(0, y): F_{1}^{+}(0, y) \cdot F_{1}^{-}(0, y)>0\right\} \text { (crossing set). } \\
& \Sigma^{e}=\left\{(0, y): F_{1}^{+}(0, y)>0 \text { and } F_{1}^{-}(0, y)<0\right\} \text { (escaping set). } \\
& \Sigma^{s}=\left\{(0, y): F_{1}^{+}(0, y)<0 \text { and } F_{1}^{-}(0, y)>0\right\} \text { (sliding set). }
\end{aligned}
$$

The boundary between the sets $\Sigma^{c}, \Sigma^{s}$ and $\Sigma^{e}$ is given by fold points. We say that $(0, y) \in \Sigma$ is a fold point for the PWL vector field (1) if $F_{1}^{-}(0, y)=0$ and $a_{12}^{-} F_{2}^{-}(0, y) \neq 0$, or $F_{1}^{+}(0, y)=0$ and $a_{12}^{+} F_{2}^{+}(0, y) \neq 0$. The PWL vector field (1) has at most two fold points. In fact, if $a_{12}^{ \pm}=0$, then the vector field does not have fold points. If $a_{12}^{ \pm} \neq 0$, then the equations $F_{1}^{ \pm}(0, y)=0$ imply the existence of two fold points.

According to the convex method of Filippov, the trajectories of $F \in \Omega^{L}$ on $\Sigma^{e} \cup \Sigma^{s}$ satisfy the equation $\dot{\mathrm{x}}=\lambda F^{-}(\mathrm{x})+(1-\lambda) F^{+}(\mathrm{x})$, where $\lambda$ is selected so that the vector field is tangent to the escaping or sliding set, i.e., $\lambda F_{1}^{-}(\mathrm{x})+$ $(1-\lambda) F_{1}^{+}(\mathrm{x})=0$.

$$
\begin{gathered}
\text { Then, for } \mathrm{x}=(0, y) \in \Sigma^{e} \cup \Sigma^{s} \text { we get } \lambda(y)=\frac{F_{1}^{+}(\mathrm{x})}{F_{1}^{+}(\mathrm{x})-F_{1}^{-}(\mathrm{x})}, \text { and so we have } \\
\dot{x}=0, \quad \dot{y}=g(y)=\frac{F_{1}^{+}(\mathrm{x}) F_{2}^{-}(\mathrm{x})-F_{1}^{-}(\mathrm{x}) F_{2}^{+}(\mathrm{x})}{F_{1}^{+}(\mathrm{x})-F_{1}^{-}(\mathrm{x})}, \quad \mathrm{x} \in \Sigma^{e} \cup \Sigma^{s} .
\end{gathered}
$$


The corresponding vector field $F^{\Sigma}(y)=(0, g(y))$ is called sliding vector field.

Here, we study PWL vector fields satisfying that the equilibrium points in the left and in the right half-planes are saddle or focus, more specifically we consider the following configurations: saddle-saddle, saddle-focus, focus-saddle, and focus-focus. We say that vector field (1) is of the kind saddle-saddle if the singularities of $F^{-}$and $F^{+}$are saddles. Analogously we define focus-saddle kind, saddle-focus kind and focus-focus kind.

We classify PWL vector fields with respect to some parameters. For the saddlesaddle case we have 4 parameters: sets on $\Sigma$ (sliding, escaping or crossing), equilibrium point (real, virtual), interception of the stable and unstable manifolds corresponding to the system $F^{-}$with $\Sigma$ and interception of the stable and unstable manifolds corresponding to the system $F^{+}$with $\Sigma$. For the saddle-focus case we have 5 parameters: we consider the first three parameters of saddle-saddle case and add two parameters: orientation and stability of the focus. For the focusfocus case we have 6 parameters: we remove the third parameter of saddle-focus case and add two parameters for the focus of system $F^{-}$: orientation and stability.

A configuration is a vector formed by the previous parameters. We define the classes $\mathcal{P}_{1}, \mathcal{P}_{2}, \mathcal{P}_{2}^{\prime}$, and $\mathcal{P}_{3}$ which are formed by saddle-saddle, saddle-focus, focus-saddle and focus-focus configurations, respectively. We define three equivalent relations $R_{1}, R_{2}$ and $R_{3}$ and study the cardinalities of quotient spaces $\mathcal{P}_{1} / R_{1}$, $\left(\mathcal{P}_{2} \cup \mathcal{P}_{2}^{\prime}\right) / R_{2}$ and $\mathcal{P}_{3} / R_{3}$. Besides, we analyze for which configurations of quotient spaces the corresponding PWL vector fields can have or not closed sliding poly-trajectories. For definition of closed sliding poly-trajectory, see Definition 5.

Remark about node case. The analysis of this case involves additional parameters which determine the relative positions of eigenspaces and $\Sigma$. It would become the paper very large and we decide consider this case in a future paper. There are many papers about PWL-systems with main goal being the search of upper bounds for the number of periodic orbits. Most of them, see [3, 4] and [5] for instance, consider systems of the kind saddle-saddle, saddle-focus and focusfocus. In our work we give necessary conditions for such configurations present sliding closed poly-trajectories.

The paper is organized as follows. In Section 2 we present the main results and introduce the notations. In Sections 3 and 4 we prove the main results. In Section 5 we present some results regarding to crossing poly-trajectories.

\section{Statement of the Main Results}

We classify the vector fields (1) with respect to the kind of equilibrium point (saddle or focus) on each half-plane and with respect to the discontinuous set (sliding, escape, crossing). Besides, if there exist equilibrium points of the kind saddle, we analyze the intersection of $\Sigma$ with the stable and instable manifolds. 


\subsection{The Saddle-Saddle Case}

We say that an equilibrium point $p$ of kind saddle is real when it belongs to the open half-plane where we have defined the corresponding linear differential system and it is called virtual if belongs to the other open half-plane. Here, we are not considering the case where the equilibrium point belongs to $\Sigma$. If the equilibrium point belongs to $\Sigma$ then it is impossible to get a closed sliding poly-trajectory and one of our purposes is to study the appearance of closed sliding poly-trajectories. The definition of poly-trajectory is given in Definition 5 .

We introduce the following notations.

(a) $\mathbf{C}, \mathbf{S}$ and $\mathbf{E}$ represent respectively the crossing, sliding and escaping sets in the line of discontinuity. So we have for example, the following combinations: C, CS, CSC. In our notation CSC means that $\Sigma$ is given by

$$
\Sigma=(-\infty, a) \cup(a, b) \cup(b,+\infty)
$$

which $(-\infty, a) \cup(b,+\infty) \subset \Sigma^{c},(a, b) \subset \Sigma^{s}$ and $a, b$ are fold points. Analogously for the other cases.

(b) $\mathbf{R}$ and $\mathbf{V}$ represent real and virtual equilibrium point, respectively. For instance, if the equilibrium point $p^{-}$of $F^{-}$is real and the equilibrium point $p^{+}$of $F^{+}$is virtual we denote $\mathbf{R V}$.

(c) $\mathbf{W}_{L}^{s}$ and $\mathbf{W}_{L}^{u}$ represent respectively the stable and unstable manifolds on the left half-plane and $\mathbf{W}_{R}^{s}$ and $\mathbf{W}_{R}^{u}$ represent respectively the stable and unstable manifolds on the right half-plane, i.e., $\mathbf{W}_{L}^{\lambda}=\mathbf{W}_{L}^{\lambda}\left(p^{-}\right) \cap \Sigma^{-}$and $\mathbf{W}_{R}^{\lambda}=\mathbf{W}_{R}^{\lambda}\left(p^{+}\right) \cap \Sigma^{+}$, for $\lambda \in\{s, u\}$.

(d) $\mathbf{F}_{L}$ and $\mathbf{F}_{R}$ represent the $y$-coordinate of the fold points of the vector fields $F^{-}$and $F^{+}$, respectively. For commodity, we call $\mathbf{F}_{L}$ and $\mathbf{F}_{R}$ fold points because the $x$-coordinate of these fold points is equal to zero.

We consider the following parameters.

$\left(n_{1}\right) 39$ possibilities for the regions on $\Sigma$ (C, S, E, CC, etc), (3+3.3+3.3.3).

$\left(n_{2}\right) 4$ possibilities for equilibrium points (RR, RV, VR, VV).

$\left(n_{3}\right)$ Relative position of the invariant manifolds in left half-plane with $\Sigma$. There are 4 possible choices for the parameter $n_{3}$ :

$$
\mathbf{W}_{L}^{s} \cap \Sigma=\varnothing, \quad \mathbf{W}_{L}^{u} \cap \Sigma=\varnothing, \quad \mathbf{W}_{L}^{s} \cap \Sigma<\mathbf{F}_{L}, \quad \mathbf{W}_{L}^{s} \cap \Sigma>\mathbf{F}_{L} .
$$

$\left(n_{4}\right)$ Relative position of the invariant manifolds in right half-plane with $\Sigma$. There are 4 possible choices for the parameter $n_{4}$ :

$$
\mathbf{W}_{R}^{s} \cap \Sigma=\varnothing, \quad \mathbf{W}_{R}^{u} \cap \Sigma=\varnothing, \quad \mathbf{W}_{R}^{s} \cap \Sigma<\mathbf{F}_{R}, \quad \mathbf{W}_{R}^{s} \cap \Sigma>\mathbf{F}_{R} .
$$

Denote

$$
\mathcal{P}_{1}=\left\{\left(n_{1}, n_{2}, n_{3}, n_{4}\right) \mid n_{1} \in\{1, . ., 39\}, n_{2}, n_{3}, n_{4} \in\{1, . ., 4\}\right\} .
$$

Definition 1. A saddle-saddle configuration is a vector $p=\left(n_{1}, n_{2}, n_{3}, n_{4}\right) \in \mathcal{P}_{1}$. 


\subsection{The Saddle-Focus (Focus-Saddle) case}

We say that an equilibrium point of kind focus is real if it belongs to the open halfplane where we have defined the corresponding linear differential system and it is called virtual otherwise. If $F^{+}\left(F^{-}\right)$has a real or virtual focus, then $F^{+}\left(F^{-}\right)$has a fold point.

The parameters $\left(n_{1}\right),\left(n_{2}\right)$ and $\left(n_{3}\right)$ introduced on subsection 2.1 are the same. Now $n_{1}=m_{1}, n_{2}=m_{2}, n_{3}=m_{3}$ and consider the new parameters.

$\left(m_{3}^{\prime}\right)$ Relative position of the invariant manifolds in right half-plane with $\Sigma$. There are 4 possible choices for the parameter $n_{3}$ :

$$
\mathbf{W}_{R}^{s} \cap \Sigma=\varnothing, \quad \mathbf{W}_{R}^{u} \cap \Sigma=\varnothing, \quad \mathbf{W}_{R}^{s} \cap \Sigma<\mathbf{F}_{R}, \quad \mathbf{W}_{R}^{s} \cap \Sigma>\mathbf{F}_{R} .
$$

$\left(m_{4}\right)$ Orientability of the focus in the right half-plane. $m_{4}=-1$ and $m_{4}=+1$, represent the counterclockwise and clockwise orientation, respectively.

$\left(m_{4}^{\prime}\right)$ Orientability of the focus in the left half-plane. $m_{4}^{\prime}=-1$ and $m_{4}^{\prime}=+1$, represent the counterclockwise and clockwise orientation, respectively.

$\left(m_{5}\right)$ Stability of the focus in the right half-plane. $m_{5}<0$ and $m_{5}>0$, represent attracting focus and repelling focus, respectively.

$\left(m_{5}^{\prime}\right)$ Stability of the focus in the left half-plane. $m_{5}^{\prime}<0$ and $m_{5}^{\prime}>0$, represent attracting focus and repelling focus, respectively.

Denote

$$
\begin{aligned}
\mathcal{P}_{2}^{1} & =\left\{\left(m_{1}, m_{2}, m_{3}, m_{4}, m_{5}\right) \mid m_{1} \in\{1, . .39\}, m_{2}, m_{3} \in\{1, . ., 4\}, m_{4}, m_{5}\right. \\
& \in\{1,2\}\} . \\
\mathcal{P}_{2}^{2} & =\left\{\left(m_{1}, m_{2}, m_{3}^{\prime}, m_{4}^{\prime}, m_{5}^{\prime}\right) \mid m_{1} \in\{1, . .39\}, m_{2}, m_{3}^{\prime} \in\{1, . ., 4\}, m_{4}^{\prime}, m_{5}^{\prime}\right. \\
& \in\{1,2\}\} .
\end{aligned}
$$

Definition 2. A saddle-focus configuration is a vector $p=\left(m_{1}, m_{2}, m_{3}, m_{4}\right.$, $\left.m_{5}\right) \in \mathcal{P}_{2}^{1} . \quad$ A focus-saddle configuration is a vector $p^{\prime}=\left(m_{1}, m_{2}, m_{3}^{\prime}, m_{4}^{\prime}, m_{5}^{\prime}\right)$ $\in \mathcal{P}_{2}^{2}$. We denote $\mathcal{P}_{2}=\mathcal{P}_{2}^{1} \cup \mathcal{P}_{2}^{2}$.

\subsection{The Focus-Focus Case}

Now $s_{1}=n_{1}, s_{2}=n_{2}$ and consider the following new parameters.

$\left(s_{3}\right)$ Orientability of the focus in the left half-plane. $s_{3}=-1$ and $s_{3}=+1$, represent the counterclockwise and clockwise orientation, respectively.

$\left(s_{4}\right)$ Stability of the focus in the left half-plane. $s_{4}<0$ and $s_{4}>0$, represent attracting focus and repelling focus, respectively. 
$\left(s_{5}\right)$ Orientability of the focus in the right half-plane. $s_{5}=-1$ and $s_{5}=+1$, represent the counterclockwise and clockwise orientation, respectively.

$\left(s_{6}\right)$ Stability of the focus in the right half-plane. $s_{6}<0$ and $s_{6}>0$, represent attracting focus and repelling focus, respectively.

Denote

$$
\begin{aligned}
\mathcal{P}_{3} & =\left\{\left(s_{1}, s_{2}, s_{3}, s_{4}, s_{5}, s_{6}\right) \mid s_{1} \in\{1, . ., 39\}, s_{2} \in\{1, . ., 4\}, s_{3}, s_{4}, s_{5}, s_{6}\right. \\
& \in\{1,2\}\} .
\end{aligned}
$$

Definition 3. A focus-focus configuration is a vector $p=\left(s_{1}, s_{2}, s_{3}, s_{4}, s_{5}, s_{6}\right)$ $\in \mathcal{P}_{3}$.

We denote $\varphi^{p}$ a flow on $\mathbb{R}^{2}$ of a PWL vector field $F=\left(F^{-}, F^{+}\right)$satisfying that the phase portrait of $F$ presents the configuration $p$.

Definition 4. Let $p, q \in \mathcal{P}_{i}, i=1,2$ or 3 be configurations and $\varphi^{p}$ a flow with configuration $p$. We say that $p$ and $q$ are related by $R_{i}$ if

(i) the configuration corresponding to the flow $\varphi^{p}( \pm t, x, y)$ is equal to $q$ or

(ii) the configuration corresponding to the flow $\bar{\varphi}(t, x, y)=f_{\lambda}\left(\varphi^{p}( \pm t, x, y)\right)$ is equal to $q$ for some $\lambda=1,2,3$, where $f_{1}(x, y)=(-x, y), f_{2}(x, y)=(x,-y)$ and $f_{3}(x, y)=(-x,-y)$.

This definition does not depend of flow $\varphi^{p}$. We have that $R_{i}$ is an equivalence relation for $i=1 . .3$, so we can define the quotient spaces $\widehat{\mathcal{P}_{1}}=\mathcal{P}_{1} / R_{1}, \widehat{\mathcal{P}_{2}}=$ $\left(\mathcal{P}_{2} \cup \mathcal{P}_{2}^{\prime}\right) / R_{2}$ and $\widehat{\mathcal{P}_{3}}=\mathcal{P}_{3} / R_{3}$. Essentially, the equivalence relations introduced above consider equal two configurations that are obtained from one another via rigid motions or changes in orientation.

Theorem 1. Consider the following items.

(a) The cardinality of set $\widehat{\mathcal{P}_{1}}$ is 22 , i.e. the set $\widehat{\mathcal{P}_{1}}$ has 22 saddle-saddle configurations.

(b) The cardinality of set $\widehat{\mathcal{P}_{2}}$ is 28 , i.e. the set $\widehat{\mathcal{P}_{2}}$ has 28 saddle-focus (or focus-saddle) configurations.

(c) The cardinality of set $\widehat{\mathcal{P}_{3}}$ is 28 , i.e. the set $\widehat{\mathcal{P}_{3}}$ has 28 focus-focus configurations.

Now we present the definition of closed poly-trajectory and a theorem that relates it with the configurations obtained in Theorem 1. Related problems about closed poly-trajectories can be found in [1,7].

Definition 5. Consider a PWL vector field $F$ in the form (1).

1. A curve $\Gamma$ is a closed poly-trajectory if $\Gamma$ is closed and the following conditions are satisfied.

- $\Gamma$ contains arcs of at lest two of the vector fields $\left.F^{-}\right|_{\Sigma^{-}},\left.F^{+}\right|_{\Sigma^{+}}$and $F^{\Sigma}$. 
- The transition between arcs of $F^{-}$and arcs of $F^{+}$happens in crossing points (and vice versa).

- The transition between arcs of $F^{-}$(or $\left.F^{+}\right)$and arcs of $F^{\Sigma}$ happens through either fold points or regular points in the sliding or escaping region, respecting orientation.

2. Let $\Gamma$ be a closed poly-trajectory of $F$. We say that

- $\Gamma$ is a closed crossing poly-trajectory if $\Gamma$ meets $\Sigma$ just in crossing points and (or) fold points.

- $\Gamma$ is a closed sliding poly-trajectory if $\Gamma$ contains at least one arc of $F^{\Sigma}$. $\Gamma$ is called closed sliding poly-trajectory of $F^{-}$if it contains arcs of $F^{-}$and does not contain arcs of $F^{+}$. Analogous to $F^{+}$.

3. Let $\Gamma$ be a closed poly-trajectory. We say that $\Gamma$ is hyperbolic if

- $\Gamma$ is a crossing poly-trajectory and $\eta^{\prime}(p) \neq 1$ where $\eta$ is the first return map defined on a segment $N$ with $p \in N \pitchfork \gamma$;

- $\Gamma$ is a closed sliding poly-trajectory and all arcs of $F^{\Sigma}$ are sliding or all are escaping.
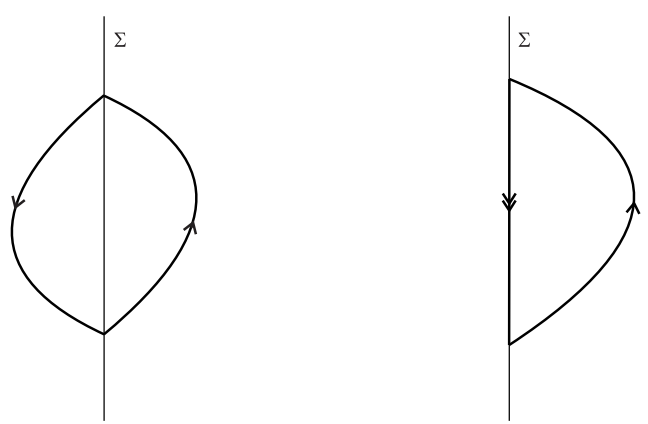

Figure 1: Closed crossing poly-trajectory and closed sliding poly-trajectory, respectively.

Theorem 2 (Non-existence of closed sliding poly-trajectories). Consider the following items.

(a) If a PWL vector field of kind saddle-saddle has a closed sliding poly-trajectory, then its configuration is the one given by line 13 of Table 1.

(b) If a PWL vector field of kind saddle-focus has a closed sliding poly-trajectory, then its configuration is given by one of lines 1, 6, 11, 17, 21 and 25-27 of Table 2.

(c) If a PWL vector field of kind focus-focus has a closed sliding poly-trajectory, then its configuration is given by one of lines 3, 6, 10-12, 17, 20 and 23-26 of Table 3. 
Theorem 3. For each of the 20 configurations listed in Theorem 2 we find one or at most two quadratic polynomials $R\left(a_{i j}^{ \pm}, b_{j}^{ \pm}\right)$and $S\left(a_{i j}^{ \pm}, b_{j}^{ \pm}\right), i, j=1,2$, whose signs determine the set of parameters for which there exist closed sliding poly-trajectories.

Each configuration depends of the parameters $\left(a_{i j}^{ \pm}\right.$and $\left.b_{j}^{ \pm}\right)$. The existence of sliding closed poly-trajectories depends of some inequalities which are related with: equilibrium position (real or virtual), the sign of derivative of the sliding vector field and the determinant of the matrices $A^{ \pm}$. Each one of these expressions is a polynomial depending on $a_{i j}^{ \pm}$and $b_{j}^{ \pm}$.

\section{Proof of Theorems 1 and 2}

In this section we proof the main results of the paper. For commodity, we say only the term configuration in the proofs of theorems 1, 2 and 3 instead of saddlesaddle configuration, saddle-focus configuration and focus-focus configuration.

\subsection{Proof of Theorem 1-(a)}

Combining the possible choices for the parameters $n_{1}, n_{2}, n_{3}, n_{4}$, we have 2496 possible configurations.

Lemma 1. If the vector field (1) is of the kind saddle-saddle, then the cardinality of set $\mathcal{P}_{1}$ is 88 , ie, the set $\mathcal{P}_{1}$ has 88 saddle-saddle configurations.

Proof. We have:

(i) If $\mathbf{W}_{\Lambda_{1}}^{\lambda_{1}} \cap \Sigma=\varnothing$ and $\mathbf{W}_{\Lambda_{2}}^{\lambda_{2}} \cap \Sigma=\varnothing$ for $\Lambda_{1} \neq \Lambda_{2} \in\{L, R\}, \lambda_{1}, \lambda_{2} \in\{s, u\}$ then $F^{\Lambda_{1}}, F^{\Lambda_{2}}$ do not have fold points. This reduces the number of configurations to 1920 .

(ii) If $\mathbf{W}_{\Lambda_{1}}^{\lambda_{1}} \cap \Sigma \neq \varnothing, \mathbf{W}_{\Lambda_{1}}^{\lambda_{2}} \cap \Sigma \neq \varnothing$ and $\mathbf{W}_{\Lambda_{2}}^{\lambda_{3}} \cap \Sigma=\varnothing$ for $\Lambda_{1} \neq \Lambda_{2} \in\{L, R\}, \lambda_{1} \neq$ $\lambda_{2}, \lambda_{3} \in\{s, u\}$ then there exist only one fold point. This fact reduces the number of configurations to 960 .

(iii) If $\mathbf{W}_{\Lambda_{1}}^{\lambda_{1}} \cap \Sigma \neq \varnothing$ and $\mathbf{W}_{\Lambda_{2}}^{\lambda_{2}} \cap \Sigma \neq \varnothing$ for all $\Lambda_{1}, \Lambda_{2} \in\{L, R\}, \lambda_{1}, \lambda_{2} \in\{s, u\}$ then there exist one or two fold points. It reduces the number of configurations to 912.

(iv) For the cases without fold points or with only one fold point, for each choose of the parameters $n_{2}, n_{3}$ and $n_{4}$, there is only one possible choice for $n_{1}$. It reduces the number of configurations to 496 .

(v) For the configurations with two fold points, with parameters $n_{2}$ assuming $\mathbf{R V}$ or VR, and $n_{3}$ and $n_{4}$ given by $\mathbf{W}_{\Lambda_{1}}^{s} \cap \Sigma>\mathrm{F}_{\Lambda_{1}}, \mathbf{W}_{\Lambda_{2}}^{s} \cap \Sigma<\mathrm{F}_{\Lambda_{2}}, \Lambda_{1}, \Lambda_{2} \in$ $\{L, R\}, \Lambda_{1} \neq \Lambda_{2}$ there is only one possible choice for $n_{1}$. The same with $n_{2}$ assuming $\mathbf{R R}$ or $\mathbf{V V}$, and $n_{3}$ and $n_{4}$ given by $\mathbf{W}_{\Lambda_{1}}^{s} \cap \Sigma>\mathrm{F}_{\Lambda_{1}}, \mathbf{W}_{\Lambda_{2}}^{s} \cap \Sigma>$ $\mathrm{F}_{\Lambda_{2}}$ or $\mathbf{W}_{\Lambda_{1}}^{s} \cap \Sigma<\mathrm{F}_{\Lambda_{1}}, \mathbf{W}_{\Lambda_{2}}^{s} \cap \Sigma<\mathrm{F}_{\Lambda_{2}}, \Lambda_{1}, \Lambda_{2} \in\{L, R\}, \Lambda_{1} \neq \Lambda_{2}$. It 


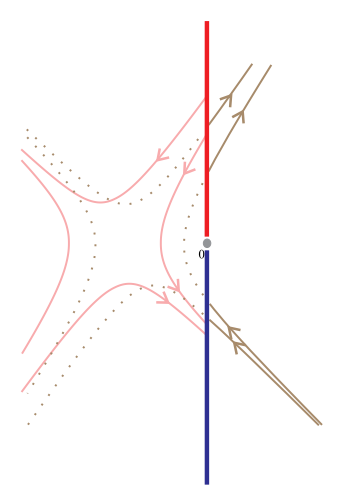

Figure 2: Lines 13 of Table 1.

reduces the number of configurations to 288 . For the other cases with two fold points, for each choose of the parameters $P_{2}, P_{3}$ and $n_{4}$ there are two possible choice for $n_{1}$. So we can to reduce the number of configurations to 88 as wanted.

Proof of Theorem 1-(a). According Lemma 1 we have that the cardinality of $\mathcal{P}_{1}$ is 88 . These configurations may be reduced to 44 configurations considering the item (i) of relation $R_{1}$, for instance the configurations

$$
\begin{array}{llll}
\mathbf{E} & \mathbf{R R} & \mathbf{W}_{L}^{u} \cap \Sigma=\varnothing & \mathbf{W}_{R}^{u} \cap \Sigma=\varnothing \\
\mathbf{S} & \mathbf{R R} & \mathbf{W}_{L}^{s} \cap \Sigma=\varnothing & \mathbf{W}_{R}^{s} \cap \Sigma=\varnothing
\end{array}
$$

are equivalents. Thus by relation $R_{1}$ we consider the lines of Table 1 . We can still reduce to 22 configurations (see Table 1). In fact, the configurations corresponding to the lines $27,28,30,31,34,35,36,37,38,39,41,42,43,40$ and 44 are equivalents to configurations corresponding to the lines $26,3,29,6,7,8,33,10,32,9,15$, $24,16,13$ and 87 respectively, by relation $R_{1}$ (use $f_{3}(x, y)=(-x,-y)$ ), and the configurations corresponding to the lines $3,6,9,10,12,16$ and 19 are equivalents to configurations $26,29,32,33,23,24$ and 25 respectively also by relation $R_{1}$ (use $\left.f_{1}(x, y)=(x,-y)\right)$.

\subsection{Proof of Theorem 1-(b)}

Combining the possible choices for the parameters $m_{1}, m_{2}, m_{3}, m_{4}, m_{5}$, we have 2496 possible configurations.

Lemma 2. If the vector field (1) is of the kind saddle-focus, then cardinality of set $\mathcal{P}_{2}$ is 112 , i.e., the set $\mathcal{P}_{2}$ has 112 saddle-focus configurations.

Proof. We have:

(i) If $\mathbf{W}_{L}^{\lambda} \cap \Sigma=\varnothing, \lambda \in\{s, u\}$, then there is only a fold point to $F$. It reduces the number of combinations to 1536 . 


\begin{tabular}{|c|c|c|c|c|c|c|}
\hline \multicolumn{7}{|c|}{ SADDLE - SADDLE } \\
\hline 1 & $\mathbf{E}$ & $\mathbf{R R}$ & $\mathbf{W}_{L}^{u} \cap \Sigma=\varnothing$ & $\mathbf{W}_{L}^{s} \cap \Sigma \neq \varnothing$ & $\mathbf{W}_{R}^{u} \cap \Sigma=\varnothing$ & $\mathbf{W}_{R}^{s} \cap \Sigma \neq \varnothing$ \\
\hline 2 & $\mathrm{C}$ & RR & $\mathbf{W}_{L}^{u} \cap \Sigma=\varnothing$ & $\mathbf{W}_{L}^{s} \cap \Sigma \neq \varnothing$ & $\mathbf{W}_{R}^{s} \cap \Sigma=\varnothing$ & $\mathbf{W}_{R}^{s} \cap \Sigma=\varnothing$ \\
\hline 3 & EC & RR & $\mathbf{W}_{L}^{u} \cap \Sigma=\varnothing$ & $\mathbf{W}_{L}^{s} \cap \Sigma \neq \varnothing$ & $\overline{\mathbf{W}_{R}^{s} \cap \Sigma>\mathbf{F}_{R}}$ & $\overline{\mathbf{W}_{R}^{u} \cap \Sigma<\mathbf{F}_{R}}$ \\
\hline 4 & $\mathbf{S}$ & $\mathbf{V V}$ & $\mathbf{W}_{L}^{u} \cap \Sigma=\varnothing$ & $\mathbf{W}_{L}^{s} \cap \Sigma \neq \varnothing$ & $\mathbf{W}_{R}^{u} \cap \Sigma=\varnothing$ & $\mathbf{W}_{R}^{s} \cap \Sigma \neq \varnothing$ \\
\hline 5 & $\mathrm{C}$ & $\mathbf{V V}$ & $\mathbf{W}_{L}^{u} \cap \Sigma=\varnothing$ & $\mathbf{W}_{L}^{s} \cap \Sigma \neq \varnothing$ & $\mathbf{W}_{R}^{s} \cap \Sigma=\varnothing$ & $\mathbf{W}_{R}^{u} \cap \Sigma \neq \varnothing$ \\
\hline 6 & SC & VV & $\mathbf{W}_{L}^{u} \cap \Sigma=\varnothing$ & $\mathbf{W}_{L}^{s} \cap \Sigma \neq \varnothing$ & $\mathbf{W}_{R}^{s} \cap \Sigma>\mathbf{F}_{R}$ & $\mathbf{W}_{R}^{u} \cap \Sigma<\mathbf{F}_{R}$ \\
\hline 7 & $\mathrm{C}$ & VR & $\mathbf{W}_{L}^{u} \cap \Sigma=\varnothing$ & $\mathbf{W}_{L}^{s} \cap \Sigma \neq \varnothing$ & $\mathbf{W}_{R}^{u} \cap \Sigma=\varnothing$ & $\mathbf{W}_{R}^{s} \cap \Sigma \neq \varnothing$ \\
\hline 8 & $\bar{S}$ & VR & $\mathbf{W}_{L}^{u} \cap \Sigma=\varnothing$ & $\mathbf{W}_{L}^{s} \cap \Sigma \neq \varnothing$ & $\mathbf{W}_{R}^{s} \cap \Sigma=\varnothing$ & $\mathbf{W}_{R}^{u} \cap \Sigma \neq \varnothing$ \\
\hline 9 & CS & VR & $\mathbf{W}_{L}^{u} \cap \Sigma=\varnothing$ & $\mathbf{W}_{L}^{s} \cap \Sigma \neq \varnothing$ & $\overline{\mathbf{W}_{R}^{s} \cap \Sigma>\mathbf{F}_{R}}$ & $\overline{\mathbf{W}_{R}^{u} \cap \Sigma<\mathbf{F}_{R}}$ \\
\hline 10 & $\mathrm{CE}$ & VR & $\mathbf{W}_{L}^{s} \cap \Sigma>\mathbf{F}_{L}$ & $\mathbf{W}_{L}^{u} \cap \Sigma<\mathbf{F}_{L}$ & $\mathbf{W}_{R}^{u} \cap \Sigma=\varnothing$ & $\mathbf{W}_{R}^{s} \cap \Sigma \neq \varnothing$ \\
\hline 11 & $\mathrm{CC}$ & $\mathbf{R R}$ & $\mathbf{W}_{L}^{s} \cap \Sigma>\mathbf{F}_{L}$ & $\mathbf{W}_{L}^{u} \cap \Sigma<\mathbf{F}_{L}$ & $\overline{\mathbf{W}_{R}^{s} \cap \Sigma<\mathbf{F}_{R}}$ & $\mathbf{W}_{R}^{u} \cap \Sigma>\mathbf{F}_{R}$ \\
\hline 12 & CEC & $\mathbf{R R}$ & $\mathbf{W}_{I}^{s} \cap \Sigma>\mathbf{F}_{L}$ & $\mathbf{W}_{I}^{u} \cap \Sigma<\mathbf{F}_{L}$ & $\mathbf{W}_{R}^{s} \cap \Sigma<\mathbf{F}_{R}$ & $\mathbf{W}_{R}^{u} \cap \Sigma>\mathbf{F}_{R}$ \\
\hline 13 & ES & $\mathbf{R V}$ & $\mathbf{W}_{L}^{s} \cap \Sigma>\mathbf{F}_{L}$ & $\mathbf{W}_{L}^{u} \cap \Sigma<\mathbf{F}_{L}$ & $\mathbf{W}_{R}^{S} \cap \Sigma<\mathbf{F}_{R}$ & $\mathbf{W}_{R}^{u} \cap \Sigma>\mathbf{F}_{R}$ \\
\hline 14 & ES & $\mathbf{R R}$ & $\mathbf{W}_{L}^{\mathrm{s}} \cap \Sigma>\mathbf{F}_{L}$ & $\mathbf{W}_{L} \cap \Sigma<\mathbf{F}_{L}$ & $\mathbf{W}_{R}^{s} \cap \Sigma>\mathbf{F}_{R}$ & $\mathbf{W}_{R}^{u} \cap \Sigma<\mathbf{F}_{R}$ \\
\hline 15 & $\mathrm{CC}$ & RV & $\mathbf{W}_{L}^{s} \cap \Sigma>\mathbf{F}_{L}$ & $\mathbf{W}_{L}^{u} \cap \Sigma<\mathbf{F}_{L}$ & $\mathbf{W}_{R}^{s} \cap \Sigma>\mathbf{F}_{R}$ & $\mathbf{W}_{R}^{u} \cap \Sigma<\mathbf{F}_{R}$ \\
\hline 16 & CEC & RV & $\mathbf{W}_{L}^{s} \cap \Sigma>\mathbf{F}_{L}$ & $\mathbf{W}_{L}^{u} \cap \Sigma<\mathbf{F}_{L}$ & $\mathbf{W}_{R}^{s} \cap \Sigma>\mathbf{F}_{R}$ & $\mathbf{W}_{R}^{s[u} \cap \Sigma<\mathbf{F}_{R}$ \\
\hline 17 & ES & $\mathbf{V V}$ & $\mathbf{W}_{L}^{s} \cap \Sigma<\mathbf{F}_{L}$ & $\mathbf{W}_{L}^{u} \cap \Sigma>\mathbf{F}_{L}$ & $\mathbf{W}_{R}^{s} \cap \Sigma<\mathbf{F}_{R}$ & $\mathbf{W}_{R}^{u} \cap \Sigma>\mathbf{F}_{R}$ \\
\hline 18 & $\overline{\mathrm{CC}}$ & $\mathbf{V V}$ & $\mathbf{W}_{L}^{s} \cap \Sigma>\mathbf{F}_{L}$ & $\mathbf{W}_{L}^{u} \cap \Sigma<\mathbf{F}_{L}$ & $\overline{\mathbf{W}_{R}^{s} \cap \Sigma<\mathbf{F}_{R}}$ & $\overline{\mathbf{W}_{R}^{u} \cap \Sigma>\mathbf{F}_{R}}$ \\
\hline 19 & CEC & $\mathbf{V V}$ & $\mathbf{W}_{L}^{s} \cap \Sigma>\mathbf{F}_{L}$ & $\mathbf{W}_{L}^{u} \cap \Sigma<\mathbf{F}_{L}$ & $\mathbf{W}_{R}^{s} \cap \Sigma<\mathbf{F}_{R}$ & $\mathbf{W}_{R}^{u} \cap \Sigma>\mathbf{F}_{R}$ \\
\hline 20 & ECS & RR & $\mathbf{W}_{L}^{s} \cap \Sigma>\mathbf{F}_{L}$ & $\overline{\mathbf{W}_{L}^{u} \cap \Sigma<\mathbf{F}_{L}}$ & $\mathbf{W}_{R}^{s} \cap \Sigma>\mathbf{F}_{R}$ & $\mathbf{W}_{R}^{u} \cap \Sigma<\mathbf{F}_{R}$ \\
\hline 21 & ECS & $\mathbf{V V}$ & $\mathbf{W}_{L}^{s} \cap \Sigma<\mathbf{F}_{L}$ & $\mathbf{W}_{L}^{u} \cap \Sigma>\mathbf{F}_{L}$ & $\mathbf{W}_{R}^{s} \cap \Sigma<\mathbf{F}_{R}$ & $\mathbf{W}_{R}^{u} \cap \Sigma>\mathbf{F}_{R}$ \\
\hline 22 & SCE & RV & $\mathbf{W}_{L}^{s} \cap \Sigma<\mathbf{F}_{L}$ & $\mathbf{W}_{L}^{u} \cap \Sigma>\mathbf{F}_{L}$ & $\overline{\mathbf{W}_{R}^{s} \cap \Sigma>\mathbf{F}_{R}}$ & $\mathbf{W}_{R}^{u} \cap \Sigma<\mathbf{F}_{R}$ \\
\hline 23 & CSC & RR & $\mathbf{W}_{L}^{s} \cap \Sigma>\mathbf{F}_{L}$ & $\mathbf{W}_{L}^{u} \cap \Sigma<\mathbf{F}_{L}$ & $\overline{\mathbf{W}_{R}^{s} \cap \Sigma<\mathbf{F}_{R}}$ & $\mathbf{W}_{R}^{u} \cap \Sigma>\mathbf{F}_{R}$ \\
\hline 24 & CSC & RV & $\mathbf{W}_{L}^{s} \cap \Sigma>\mathbf{F}_{L}$ & $\mathbf{W}_{L}^{u} \cap \Sigma<\mathbf{F}_{L}$ & $\mathbf{W}_{R}^{s} \cap \Sigma>\mathbf{F}_{R}$ & $\mathbf{W}_{R}^{u} \cap \Sigma<\mathbf{F}_{R}$ \\
\hline 25 & CSC & $\mathbf{V V}$ & $\mathbf{W}_{L}^{s} \cap \Sigma>\mathbf{F}_{L}$ & $\mathbf{W}_{L}^{u} \cap \Sigma<\mathbf{F}_{L}$ & $\mathbf{W}_{R}^{s} \cap \Sigma<\mathbf{F}_{R}$ & $\mathbf{W}_{R}^{u} \cap \Sigma>\mathbf{F}_{R}$ \\
\hline 26 & $\mathrm{CE}$ & RR & $\mathbf{W}_{L}^{u} \cap \Sigma=\varnothing$ & $\mathbf{W}_{L}^{s} \cap \Sigma \neq \varnothing$ & $\mathbf{W}_{R}^{s} \cap \Sigma<\mathbf{F}_{R}$ & $\mathbf{W}_{R}^{u} \cap \Sigma>\mathbf{F}_{R}$ \\
\hline 27 & EC & $\mathbf{R R}$ & $\mathbf{W}_{L}^{s} \cap \Sigma>\mathbf{F}_{L}$ & $\mathbf{W}_{L}^{u} \cap \Sigma<\mathbf{F}_{L}$ & $\mathbf{W}_{R}^{u} \cap \Sigma=\varnothing$ & $\mathbf{W}_{R}^{s} \cap \Sigma \neq \varnothing$ \\
\hline 28 & $\overline{C E}$ & $\mathbf{R R}$ & $\mathbf{W}_{L}^{s} \cap \Sigma<\mathbf{F}_{L}$ & $\mathbf{W}_{L}^{u} \cap \Sigma>\mathbf{F}_{L}$ & $\mathbf{W}_{R}^{u} \cap \Sigma=\varnothing$ & $\mathbf{W}_{R}^{s} \cap \Sigma \neq \varnothing$ \\
\hline 29 & $\mathrm{CS}$ & $\mathbf{V V}$ & $\mathbf{W}_{L}^{u} \cap \Sigma=\varnothing$ & $\mathbf{W}_{L}^{s} \cap \Sigma \neq \varnothing$ & $\mathbf{W}_{R}^{s} \cap \Sigma<\mathbf{F}_{R}$ & $\mathbf{W}_{R}^{u} \cap \Sigma>\mathbf{F}_{R}$ \\
\hline 30 & $\overline{S C}$ & $\mathbf{V V}$ & $\mathbf{W}_{L}^{s} \cap \Sigma>\mathbf{F}_{L}$ & $\mathbf{W}_{L}^{u} \cap \Sigma<\mathbf{F}_{L}$ & $\mathbf{W}_{R}^{u} \cap \Sigma=\varnothing$ & $\mathbf{W}_{R}^{s} \cap \Sigma \neq \varnothing$ \\
\hline 31 & CS & $\mathbf{V V}$ & $\mathbf{W}_{L}^{s} \cap \Sigma<\mathbf{F}_{L}$ & $\mathbf{W}_{L}^{u} \cap \Sigma>\mathbf{F}_{L}$ & $\mathbf{W}_{R}^{u} \cap \Sigma=\varnothing$ & $\mathbf{W}_{R}^{s} \cap \Sigma \neq \varnothing$ \\
\hline 32 & SC & VR & $\mathbf{W}_{L}^{u} \cap \Sigma=\varnothing$ & $\mathbf{W}_{L}^{s} \cap \Sigma \neq \varnothing$ & $\mathbf{W}_{R}^{s} \cap \Sigma<\mathbf{F}_{R}$ & $\mathbf{W}_{R}^{u} \cap \Sigma>\mathbf{F}_{R}$ \\
\hline 33 & EC & VR & $\mathbf{W}_{L}^{s} \cap \Sigma<\mathbf{F}_{L}$ & $\mathbf{W}_{L}^{u} \cap \Sigma>\mathbf{F}_{L}$ & $\mathbf{W}_{R}^{u} \cap \Sigma=\varnothing$ & $\mathbf{W}_{R}^{s} \cap \Sigma \neq \varnothing$ \\
\hline 34 & $\mathrm{C}$ & RV & $\mathbf{W}_{L}^{u} \cap \Sigma=\varnothing$ & $\mathbf{W}_{L}^{s} \cap \Sigma \neq \varnothing$ & $\mathbf{W}_{R}^{u} \cap \Sigma=\varnothing$ & $\mathbf{W}_{R}^{s} \cap \Sigma \neq \varnothing$ \\
\hline 35 & $\bar{E}$ & RV & $\mathbf{W}_{L}^{u} \cap \Sigma=\varnothing$ & $\mathbf{W}_{L}^{s} \cap \Sigma \neq \varnothing$ & $\mathbf{W}_{R}^{s} \cap \Sigma=\varnothing$ & $\mathbf{W}_{R}^{u} \cap \Sigma \neq \varnothing$ \\
\hline 36 & $\mathrm{CE}$ & RV & $\mathbf{W}_{L}^{u} \cap \Sigma=\varnothing$ & $\mathbf{W}_{L}^{s} \cap \Sigma \neq \varnothing$ & $\mathbf{W}_{R}^{S} \cap \Sigma>\mathbf{F}_{R}$ & $\mathbf{W}_{R}^{u} \cap \Sigma<\mathbf{F}_{R}$ \\
\hline 37 & EC & RV & $\mathbf{W}_{L}^{u} \cap \Sigma=\varnothing$ & $\mathbf{W}_{L}^{s} \cap \Sigma \neq \varnothing$ & $\mathbf{W}_{R}^{s} \cap \Sigma<\mathbf{F}_{R}$ & $\mathbf{W}_{R}^{u} \cap \Sigma>\mathbf{F}_{R}$ \\
\hline 38 & CS & RV & $\mathbf{W}_{L}^{s} \cap \Sigma>\mathbf{F}_{L}$ & $\mathbf{W}_{L}^{u} \cap \Sigma<\mathbf{F}_{L}$ & $\mathbf{W}_{R}^{u} \cap \Sigma=\varnothing$ & $\mathbf{W}_{R}^{s} \cap \Sigma \neq \varnothing$ \\
\hline 39 & $\overline{S C}$ & RV & $\mathbf{W}_{L}^{s} \cap \Sigma<\mathbf{F}_{L}$ & $\mathbf{W}_{L}^{u} \cap \Sigma>\mathbf{F}_{L}$ & $\mathbf{W}_{R}^{u} \cap \Sigma=\varnothing$ & $\mathbf{W}_{R}^{s} \cap \Sigma \neq \varnothing$ \\
\hline 40 & SE & VR & $\mathbf{W}_{L}^{s} \cap \Sigma>\mathbf{F}_{L}$ & $\mathbf{W}_{L}^{u} \cap \Sigma<\mathbf{F}_{L}$ & $\mathbf{W}_{R}^{s} \cap \Sigma<\mathbf{F}_{R}$ & $\mathbf{W}_{R}^{u} \cap \Sigma>\mathbf{F}_{R}$ \\
\hline 41 & $\mathrm{CC}$ & VR & $\mathbf{W}_{L}^{s} \cap \Sigma>\mathbf{F}_{L}$ & $\overline{\mathbf{W}_{L}^{u} \cap \Sigma<\mathbf{F}_{L}}$ & $\mathbf{W}_{R}^{s} \cap \Sigma>\mathbf{F}_{R}$ & $\mathbf{W}_{R}^{u} \cap \Sigma<\mathbf{F}_{R}$ \\
\hline 42 & CEC & VR & $\mathbf{W}_{L}^{s} \cap \Sigma>\mathbf{F}_{L}$ & $\mathbf{W}_{L}^{u} \cap \Sigma<\mathbf{F}_{L}$ & $\mathbf{W}_{R}^{s} \cap \Sigma>\mathbf{F}_{R}$ & $\mathbf{W}_{R}^{u} \cap \Sigma<\mathbf{F}_{R}$ \\
\hline 43 & CSC & VR & $\mathbf{W}_{L}^{s} \cap \Sigma>\mathbf{F}_{L}$ & $\mathbf{W}_{L}^{u} \cap \Sigma<\mathbf{F}_{L}$ & $\mathbf{W}_{R}^{s} \cap \Sigma>\mathbf{F}_{R}$ & $\mathbf{W}_{R}^{u} \cap \Sigma<\mathbf{F}_{R}$ \\
\hline 44 & SCE & VR & $\mathbf{W}_{L}^{s} \cap \Sigma>\mathbf{F}_{L}$ & $\mathbf{W}_{L}^{u} \cap \Sigma<\mathbf{F}_{L}$ & $\mathbf{W}_{R}^{s} \cap \Sigma<\mathbf{F}_{R}$ & $\mathbf{W}_{R}^{u} \cap \Sigma>\mathbf{F}_{R}$ \\
\hline
\end{tabular}

Table 1: Possible configurations for the saddle-saddle case using the item (i) of relation $R_{1}$. The lines $1-22$ represent the configurations of Theorem 1-(a). The highlighted line presents the necessary configuration to the existence of closed sliding poly-trajectories. 
(ii) If $\mathbf{W}_{L}^{s} \cap \Sigma \neq \varnothing$ and $\mathbf{W}_{L}^{u} \cap \Sigma \neq \varnothing$, then there exist one or two fold points. It reduces the number of combinations to 1440 .

(iii) For the case with only one fold point, for each choose of the parameters $m_{2}, m_{3}, m_{4}$ and $m_{5}$, there is only one possible choice for $m_{1}$. It reduces the number of combinations to 928 .

(iv) For the configurations with two fold points, for parameters $m_{2}$ assuming $\mathbf{R R}$ or $\mathbf{R V}$, and $m_{3}, m_{4}$ and $m_{5}$ given by $\mathbf{W}_{L}^{s} \cap \Sigma<\mathrm{F}_{L},-,>0$, or $\mathbf{W}_{L}^{s} \cap \Sigma<$ $\mathrm{F}_{L,}-,<0$, or $\mathbf{W}_{L}^{s} \cap \Sigma>\mathrm{F}_{L},+,>0$, or $\mathbf{W}_{L}^{s} \cap \Sigma>\mathrm{F}_{L,}+,<0$, there is only one possible choice for $m_{1}$. The same for $m_{2}$ assuming $\mathbf{R} \mathbf{R}$ or $\mathbf{R V}, m_{3}$, $m_{4}$ and $m_{5}$ given by $\mathbf{W}_{L}^{s} \cap \Sigma>\mathrm{F}_{L},-,>0$, or $\mathbf{W}_{L}^{s} \cap \Sigma>\mathrm{F}_{L},-,<0$, or $\mathbf{W}_{L}^{s} \cap \Sigma<\mathrm{F}_{L},+,>0$, or $\mathbf{W}_{L}^{s} \cap \Sigma<\mathrm{F}_{L},+,<0$. It reduces the number of combinations to 512. For the other cases with two fold points, for each choose of the parameters $m_{2}, m_{3} m_{4}$ and $m_{5}$ there are two possible choice for $m_{1}$. So we can reduce the number combinations to 112 as desired.

With similar tools used in the proof of Lemma 2, we prove the following lemma.

Lemma 3. If the vector field (1) is of the kind focus-saddle, then the cardinality of set $\mathcal{P}_{2}$ is 112 .

Proof of Theorem 1-(b). These 224 cases given in Lemmas 2,3 may be reduced to 112 configurations considering that the phase portraits corresponding to the saddle-focus case are equivalents to the focus-saddle case by item (ii) of relation $R_{2}$ (use $f_{3}(x, y)=(-x,-y)$ ). Without loss of generality, we consider the saddlefocus case. The 112 configurations corresponding the saddle-focus case given in Lemma 2 may be reduced to 56 considering the item (i) of relation $R_{2}$ (see Table 2). Moreover, the lines 29, 30, 31, 32, 33, 34, 35, 36, 39, 41, 40, 44, 46, 45, 47, 49, $48,52,54,53,20,21,42,43,50,51,55$ and 56 are equivalents to the 28 first lines of Table 2 respectively, by item (ii) of relation $R_{2}$ (use $f_{1}(x, y)=(x,-y)$ ).
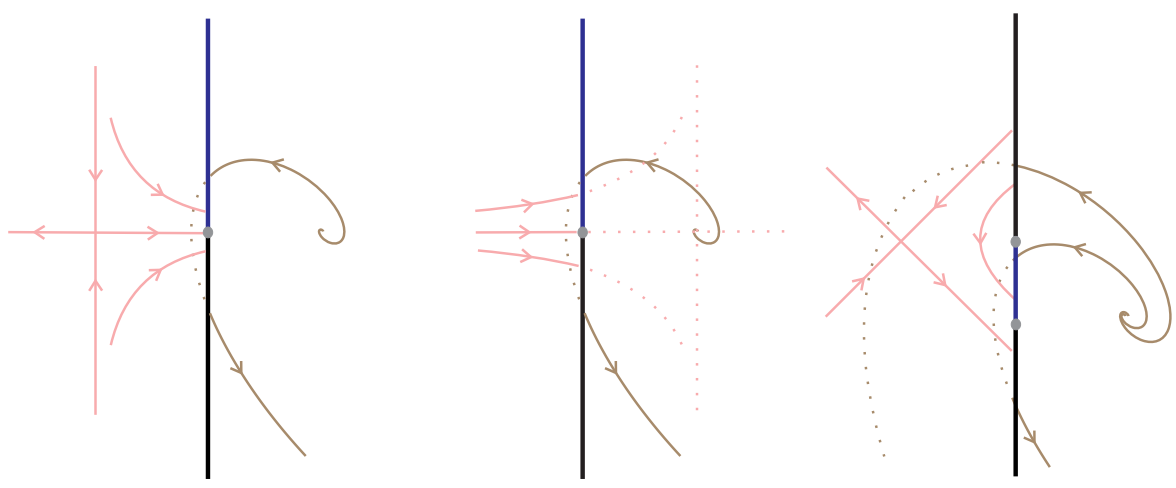

Figure 3: Lines 1, 6 and 11 of Table 2. 


\begin{tabular}{|c|c|c|c|c|c|c|}
\hline \multicolumn{7}{|c|}{ SADDLE - FOCUS } \\
\hline 1 & $\mathrm{SC}$ & $\overline{\mathbf{R R}}$ & $\mathbf{W}_{L}^{S} \cap \Sigma=\varnothing$ & $\mathbf{W}_{L}^{u} \cap \Sigma \neq \varnothing$ & $n_{4}=-1$ & $m_{5}>0$ \\
\hline 2 & $\mathrm{CE}$ & $\mathbf{R}$ & $\mathbf{W}_{L}^{u} \cap \Sigma=\varnothing$ & $\mathbf{W}_{L}^{s} \cap \Sigma \neq \varnothing$ & $m_{4}=-1$ & $m_{5}>0$ \\
\hline 3 & $\overline{\mathrm{SC}}$ & $\overline{\mathbf{R V}}$ & $\mathbf{W}_{L}^{S} \cap \Sigma=\varnothing$ & $\mathbf{W}_{L}^{u} \cap \Sigma \neq \varnothing$ & $m_{4}=-1$ & $\overline{m_{5}>0}$ \\
\hline 4 & $\overline{\mathrm{CE}}$ & RV & $\overline{\mathbf{W}}_{L}^{u} \cap \Sigma=\varnothing$ & $\mathbf{W}_{I}^{s} \cap \Sigma \neq \varnothing$ & $m_{4}=-1$ & $\overline{m_{5}>0}$ \\
\hline 5 & $\overline{C E}$ & VR & $\overline{\mathbf{W}_{L}^{s} \cap \Sigma=\varnothing}$ & $\mathbf{W}_{L}^{u} \cap \Sigma \neq \varnothing$ & $m_{4}=-1$ & $m_{5}>0$ \\
\hline 6 & SC & $\overline{V R}$ & $\mathbf{W}_{L}^{u} \cap \Sigma=\varnothing$ & $\mathbf{W}_{L}^{s} \cap \Sigma \neq \varnothing$ & $m_{4}=-1$ & $m_{5}>0$ \\
\hline 7 & $\mathrm{CE}$ & VV & $\mathbf{W}_{L}^{s} \cap \Sigma=\varnothing$ & $\mathbf{W}_{L}^{u} \cap \Sigma \neq \varnothing$ & $m_{4}=-1$ & $m_{5}>0$ \\
\hline 8 & SC & $\overline{\mathbf{V V}}$ & $\mathbf{W}_{L}^{u} \cap \Sigma=\varnothing$ & $\mathbf{W}_{L}^{s} \cap \Sigma \neq \varnothing$ & $m_{4}=-1$ & $m_{5}>0$ \\
\hline 9 & $\mathrm{CC}$ & $\mathbf{R R}$ & $\mathbf{W}_{L}^{s} \cap \Sigma>\mathbf{F}_{L}$ & $\mathbf{W}_{L}^{u} \cap \Sigma<\mathbf{F}_{L}$ & $m_{4}=-1$ & $m_{5}>0$ \\
\hline 10 & CEC & RR & $\mathbf{W}_{L}^{s} \cap \Sigma>\mathbf{F}_{L}$ & $\mathbf{W}_{L}^{u} \cap \Sigma<\mathbf{F}_{L}$ & $m_{4}=-1$ & $m_{5}>0$ \\
\hline 11 & $\mathrm{CSC}$ & $\overline{\mathbf{R R}}$ & $\mathbf{W}_{L}^{s} \cap \Sigma>\mathbf{F}_{L}$ & $\mathbf{W}_{L}^{u} \cap \Sigma<\mathbf{F}_{L}$ & $m_{4}=-1$ & $m_{5}>0$ \\
\hline 12 & $\mathrm{CC}$ & $\mathbf{R V}$ & $\mathbf{W}_{L}^{s} \cap \Sigma>\mathbf{F}_{L}$ & $\mathbf{W}_{L}^{u} \cap \Sigma<\mathbf{F}_{L}$ & $m_{4}=-1$ & $m_{5}>0$ \\
\hline 13 & CEC & $\mathbf{R V}$ & $\mathbf{W}_{L}^{s} \cap \Sigma>\mathbf{F}_{L}$ & $\mathbf{W}_{L}^{u} \cap \Sigma<\mathbf{F}_{L}$ & $m_{4}=-1$ & $m_{5}>0$ \\
\hline 14 & $\mathrm{CSC}$ & $\overline{\mathbf{R V}}$ & $\mathbf{W}_{L}^{S} \cap \sum>\mathbf{F}_{L}$ & $\mathbf{W}_{L}^{u} \cap \Sigma<\mathbf{F}_{L}$ & $m_{4}=-1$ & $m_{5}>0$ \\
\hline 15 & $\mathrm{CC}$ & VR & $\mathbf{W}_{L}^{s} \cap \Sigma<\mathbf{F}_{L}$ & $\mathbf{W}_{L}^{u} \cap \Sigma>\mathbf{F}_{L}$ & $m_{4}=-1$ & $m_{5}>0$ \\
\hline 16 & CEC & VR & $\mathbf{W}_{L}^{s} \cap \Sigma<\mathbf{F}_{L}$ & $\mathbf{W}_{L}^{u} \cap \Sigma>\mathbf{F}_{L}$ & $n_{4}=-1$ & $m_{5}>0$ \\
\hline 17 & $\mathrm{CSC}$ & VR & $\mathbf{W}_{L}^{S} \cap \Sigma<\mathbf{F}_{L}$ & $\mathbf{W}_{L}^{u} \cap \Sigma>\mathbf{F}_{L}$ & $m_{4}=-1$ & $m_{5}>0$ \\
\hline 18 & $\mathrm{CC}$ & VV & $\mathbf{W}_{L}^{s} \cap \Sigma<\mathbf{F}_{L}$ & $\mathbf{W}_{L}^{u} \cap \Sigma>\mathbf{F}_{L}$ & $m_{4}=-1$ & $m_{5}>0$ \\
\hline 19 & CEC & $\overline{\mathrm{VV}}$ & $\mathbf{W}_{L}^{s} \cap \Sigma<\mathbf{F}_{L}$ & $\mathbf{W}_{L}^{u} \cap \Sigma>\mathbf{F}_{L}$ & $m_{4}=-1$ & $m_{5}>0$ \\
\hline 20 & $\mathrm{CSC}$ & $\overline{\mathrm{VV}}$ & $\mathbf{W}_{L}^{S} \cap \Sigma<\mathbf{F}_{L}$ & $\mathbf{W}_{L}^{u} \cap \Sigma>\mathbf{F}_{L}$ & $m_{4}=-1$ & $m_{5}>0$ \\
\hline 21 & $\mathrm{ES}$ & $\overline{\mathbf{R R}}$ & $\mathbf{W}_{L}^{s} \cap \Sigma>\mathbf{F}_{L}$ & $\mathbf{W}_{L}^{u} \cap \Sigma<\mathbf{F}_{L}$ & $n_{4}=+1$ & $m_{5}>0$ \\
\hline 22 & ECS & RR & $\mathbf{W}_{L}^{s} \cap \Sigma>\mathbf{F}_{L}$ & $\mathbf{W}_{L}^{u} \cap \Sigma<\mathbf{F}_{L}$ & $m_{4}=+1$ & $m_{5}>0$ \\
\hline 23 & $\mathrm{SE}$ & RV & $\mathbf{W}_{L}^{S} \cap \Sigma<\mathbf{F}_{L}$ & $\mathbf{W}_{L}^{S} \cap \Sigma>\mathbf{F}_{L}$ & $m_{4}=-1$ & $m_{5}>0$ \\
\hline 24 & SCE & $\mathbf{R V}$ & $\mathbf{W}_{L}^{\mathbf{s}} \cap \Sigma<\mathbf{F}_{L}$ & $\mathbf{W}_{L}^{u} \cap \Sigma>\mathbf{F}_{L}$ & $n_{4}=-1$ & $m_{5}>0$ \\
\hline 25 & SE & VR & $\mathbf{W}_{L}^{s} \cap \Sigma>\mathbf{F}_{L}$ & $\mathbf{W}_{L}^{u} \cap \Sigma<\mathbf{F}_{L}$ & $m_{4}=-1$ & $m_{5}>0$ \\
\hline 26 & SCE & VR & $\mathbf{W}_{L}^{s} \cap \Sigma>\mathbf{F}_{L}$ & $\mathbf{W}_{L}^{u} \cap \Sigma<\mathbf{F}_{L}$ & $m_{4}=-1$ & $m_{5}>0$ \\
\hline 27 & $\overline{S E}$ & VV & $\mathbf{W}_{L}^{S} \cap \Sigma>\mathbf{F}_{L}$ & $\mathbf{W}_{L}^{u} \cap \Sigma<\mathbf{F}_{L}$ & $m_{4}=-1$ & $m_{5}>0$ \\
\hline 28 & SCE & VV & $\mathbf{W}_{L}^{s} \cap \Sigma>\mathbf{F}_{L}$ & $\mathbf{W}_{L}^{u} \cap \Sigma<\mathbf{F}_{L}$ & $m_{4}=-1$ & $m_{5}>0$ \\
\hline 29 & $\mathrm{CS}$ & $\overline{\mathbf{R R}}$ & $\mathbf{W}_{L}^{s} \cap \Sigma=\varnothing$ & $\mathbf{W}_{L}^{u} \cap \Sigma \neq \varnothing$ & $m_{4}=+1$ & $m_{5}>0$ \\
\hline 30 & EC & RR & $\mathbf{W}_{L}^{u} \cap \Sigma=\varnothing$ & $\mathbf{W}_{L}^{S} \cap \Sigma \neq \varnothing$ & $m_{4}=+1$ & $m_{5}>0$ \\
\hline 31 & CS & RV & $\mathbf{W}_{L}^{S} \cap \Sigma=\varnothing$ & $\mathbf{W}_{L}^{u} \cap \Sigma \neq \varnothing$ & $m_{4}=+1$ & $m_{5}>0$ \\
\hline 32 & EC & $\mathbf{R V}$ & $\mathbf{W}_{L}^{u} \cap \Sigma=\varnothing$ & $\mathbf{W}_{L}^{s} \cap \Sigma \neq \varnothing$ & $m_{4}=+1$ & $m_{5}>0$ \\
\hline 33 & EC & VR & $\mathbf{W}_{L}^{S} \cap \Sigma=\varnothing$ & $\mathbf{W}_{L}^{u} \cap \Sigma \neq \varnothing$ & $m_{4}=+1$ & $m_{5}>0$ \\
\hline 34 & $\mathrm{CS}$ & $\overline{\text { VR }}$ & $\mathbf{W}_{L}^{u} \cap \Sigma=\varnothing$ & $\mathbf{W}_{L}^{s} \cap \Sigma \neq \varnothing$ & $m_{4}=+1$ & $m_{5}>0$ \\
\hline 35 & EC & $\overline{\text { VV }}$ & $\mathbf{W}_{L}^{s} \cap \Sigma=\varnothing$ & $\mathbf{W}_{L}^{u} \cap \Sigma \neq \varnothing$ & $m_{4}=+1$ & $m_{5}>0$ \\
\hline 36 & $\mathrm{CS}$ & VV & $\mathbf{W}_{L}^{u} \cap \Sigma=\varnothing$ & $\mathbf{W}_{L}^{s} \cap \Sigma \neq \varnothing$ & $m_{4}=+1$ & $m_{5}>0$ \\
\hline 37 & $\mathrm{SE}$ & $\overline{\mathbf{R R}}$ & $\mathbf{W}_{L}^{S} \cap \Sigma<\mathbf{F}_{L}$ & $\mathbf{W}_{L}^{u} \cap \Sigma>\mathbf{F}_{L}$ & $m_{4}=-1$ & $m_{5}>0$ \\
\hline 38 & SCE & $\overline{\mathbf{R R}}$ & $\mathbf{W}_{L}^{s} \cap \Sigma<\mathbf{F}_{L}$ & $\mathbf{W}_{L}^{u} \cap \Sigma>\mathbf{F}_{L}$ & $m_{4}=-1$ & $m_{5}>0$ \\
\hline 39 & CC & RR & $\mathbf{W}_{L}^{s} \cap \Sigma<\mathbf{F}_{L}$ & $\mathbf{W}_{L}^{u} \cap \Sigma>\mathbf{F}_{L}$ & $m_{4}=+1$ & $m_{5}>0$ \\
\hline 40 & $\mathrm{CSC}$ & $\overline{\mathbf{R R}}$ & $\mathbf{W}_{L}^{\mathbf{s}} \cap \Sigma<\mathbf{F}_{L}$ & $\mathbf{W}_{L}^{u} \cap \Sigma>\mathbf{F}_{L}$ & $m_{4}=+1$ & $m_{5}>0$ \\
\hline 41 & CEC & $\overline{\mathbf{R R}}$ & $\mathbf{W}_{L}^{s} \cap \Sigma<\mathbf{F}_{L}$ & $\mathbf{W}_{L}^{u} \cap \Sigma>\mathbf{F}_{L}$ & $m_{4}=+1$ & $n_{5}>0$ \\
\hline 42 & ES & $\mathbf{R V}$ & $\mathbf{W}_{L}^{s} \cap \Sigma>\mathbf{F}_{L}$ & $\mathbf{W}_{L}^{u} \cap \Sigma<\mathbf{F}_{L}$ & $m_{4}=+1$ & $n_{5}>0$ \\
\hline 43 & ECS & $\mathbf{R V}$ & $\mathbf{W}_{L}^{s} \cap \Sigma>\mathbf{F}_{L}$ & $\mathbf{W}_{L}^{u} \cap \Sigma<\mathbf{F}_{L}$ & $m_{4}=+1$ & $m_{5}>0$ \\
\hline 44 & $\mathrm{CC}$ & $\mathbf{R V}$ & $\mathbf{W}_{L}^{s} \cap \Sigma<\mathbf{F}_{L}$ & $\mathbf{W}_{L}^{u} \cap \Sigma>\mathbf{F}_{L}$ & $m_{4}=+1$ & $m_{5}>0$ \\
\hline 45 & CSC & RV & $\mathbf{W}_{L}^{s} \cap \Sigma<\mathbf{F}_{L}$ & $\mathbf{W}_{L}^{u} \cap \Sigma>\mathbf{F}_{L}$ & $m_{4}=+1$ & $m_{5}>0$ \\
\hline 46 & CEC & $\mathbf{R V}$ & $\mathbf{W}_{L}^{S} \cap \Sigma<\mathbf{F}_{L}$ & $\mathbf{W}_{L}^{u} \cap \Sigma>\mathbf{F}_{L}$ & $m_{4}=+1$ & $m_{5}>0$ \\
\hline 47 & $\mathrm{CC}$ & VR & $\mathbf{W}_{L}^{s} \cap \Sigma>\mathbf{F}_{L}$ & $\mathbf{W}_{L}^{u} \cap \Sigma<\mathbf{F}_{L}$ & $m_{4}=+1$ & $m_{5}>0$ \\
\hline 48 & $\mathrm{CSC}$ & $\overline{\text { VR }}$ & $\mathbf{W}_{L}^{s} \cap \Sigma>\mathbf{F}_{L}$ & $\mathbf{W}_{L}^{u} \cap \Sigma<\mathbf{F}_{L}$ & $m_{4}=+1$ & $m_{5}>0$ \\
\hline 49 & CEC & $\overline{\text { VR }}$ & $\mathbf{W}_{L}^{S} \cap \sum>\mathbf{F}_{L}$ & $\mathbf{W}_{L}^{u} \cap \Sigma<\mathbf{F}_{L}$ & $m_{4}=+1$ & $m_{5}>0$ \\
\hline 50 & ES & VR & $\mathbf{W}_{L}^{s} \cap \Sigma<\mathbf{F}_{L}$ & $\mathbf{W}_{L}^{u} \cap \Sigma>\mathbf{F}_{L}$ & $m_{4}=+1$ & $m_{5}>0$ \\
\hline 51 & ECS & VR & $\mathbf{W}_{L}^{s} \cap \Sigma<\mathbf{F}_{L}$ & $\mathbf{W}_{L}^{u} \cap \Sigma>\mathbf{F}_{L}$ & $m_{4}=+1$ & $m_{5}>0$ \\
\hline 52 & $\mathrm{CC}$ & VV & $\mathbf{W}_{L}^{S} \cap \Sigma>\mathbf{F}_{L}$ & $\mathbf{W}_{L}^{u} \cap \Sigma<\mathbf{F}_{L}$ & $m_{4}=+1$ & $m_{5}>0$ \\
\hline 53 & CSC & VV & $\mathbf{W}_{L}^{s} \cap \Sigma>\mathbf{F}_{L}$ & $\mathbf{W}_{L}^{u} \cap \Sigma<\mathbf{F}_{L}$ & $m_{4}=+1$ & $m_{5}>0$ \\
\hline 54 & CEC & VV & $\mathbf{W}_{L}^{s} \cap \Sigma>\mathbf{F}_{L}$ & $\mathbf{W}_{L}^{u} \cap \Sigma<\mathbf{F}_{L}$ & $m_{4}=+1$ & $m_{5}>0$ \\
\hline 55 & EC & VV & $\mathbf{W}_{L}^{s} \cap \Sigma<\mathbf{F}_{L}$ & $\mathbf{W}_{L}^{u} \cap \Sigma>\mathbf{F}_{L}$ & $m_{4}=+1$ & $m_{5}>0$ \\
\hline 56 & ECS & VV & $\mathbf{W}_{L}^{s} \cap \Sigma<\mathbf{F}_{L}$ & $\mathbf{W}_{L}^{u} \cap \Sigma>\mathbf{F}_{L}$ & $m_{4}=+1$ & $m_{5}>0$ \\
\hline
\end{tabular}

Table 2: Possible configurations for the saddle-focus case using the item (i) of relation $R_{2}$. The lines $1-28$ represent the configurations of Theorem 1-(b). The highlighted lines present the necessary configurations to the existence of closed sliding poly-trajectories. 

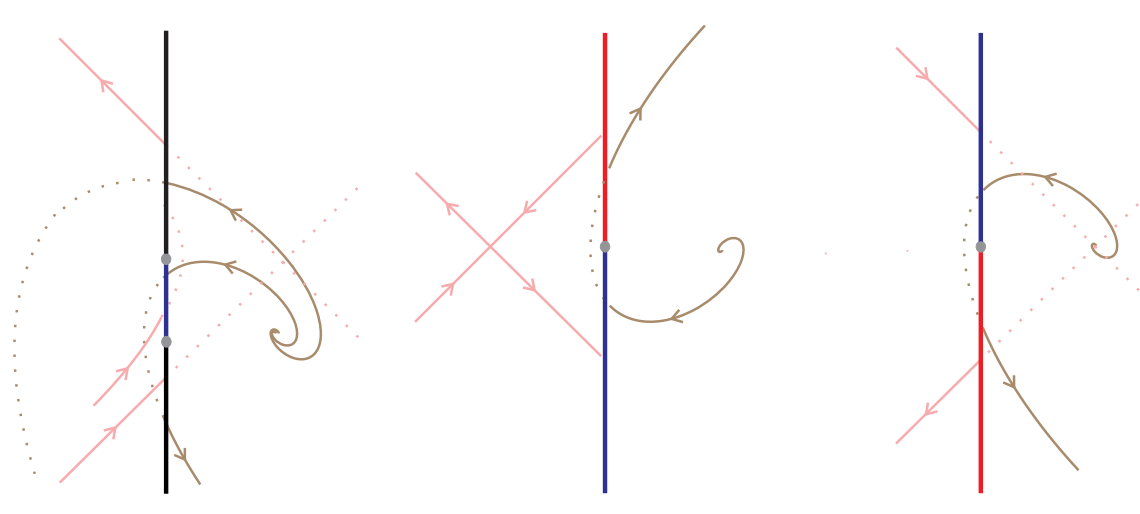

Figure 4: Lines 17, 21 and 25 of Table 2.
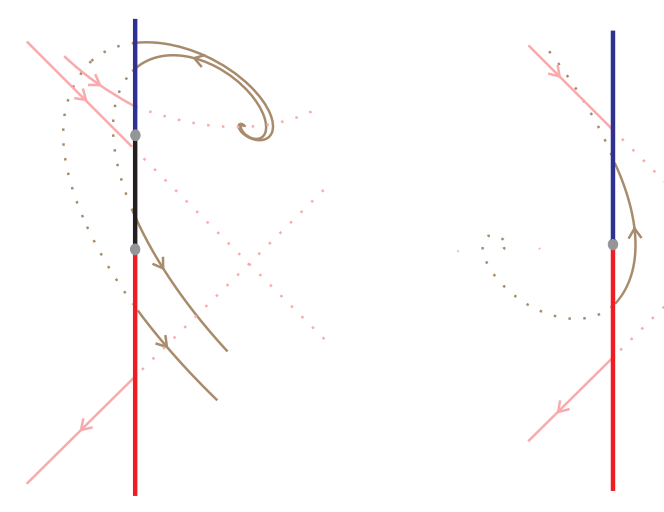

Figure 5: Lines 26 and 27 of Table 2.

\subsection{Proof of Theorem 1-(c)}

Combining the possible choices for the parameters $s_{1}, s_{2}, s_{3}, s_{4}, s_{5}$ and $s_{6}$, we have 2496 possible configurations.

Lemma 4. If the vector field (1) is of the kind focus-focus, then the cardinality of set $\mathcal{P}_{3}$ is 160 , i.e., the set $\mathcal{P}_{3}$ has 160 focus-focus configurations. These configurations are listed in Table 3.

Proof. We have

(i) As we said before, $\left(F^{-}, F^{+}\right)$has a fold point. It reduces the number of configurations to 2304 .

(ii) For the cases with only one fold point, for each choose of the parameters $s_{2}, s_{3}, s_{4}, s_{5}$ and $s_{6}$, there is only one possible choice for $s_{1}$. It reduces the number of configurations to 1792 .

(iii) For the configurations with two fold points, for parameters $s_{2}$ assuming $\mathbf{R R}, \mathbf{R V}, \mathbf{V R}$ or $\mathbf{V V}$, and $s_{3}, s_{4}, s_{5}$ and $s_{6}$ given by $+1,>0,-,>0$, or $+1,<$ $0,-,>0$, or $-1,>0,+,>0$, or $-1,<0,+,>0$, or $-1,>0,+,<0$, or 

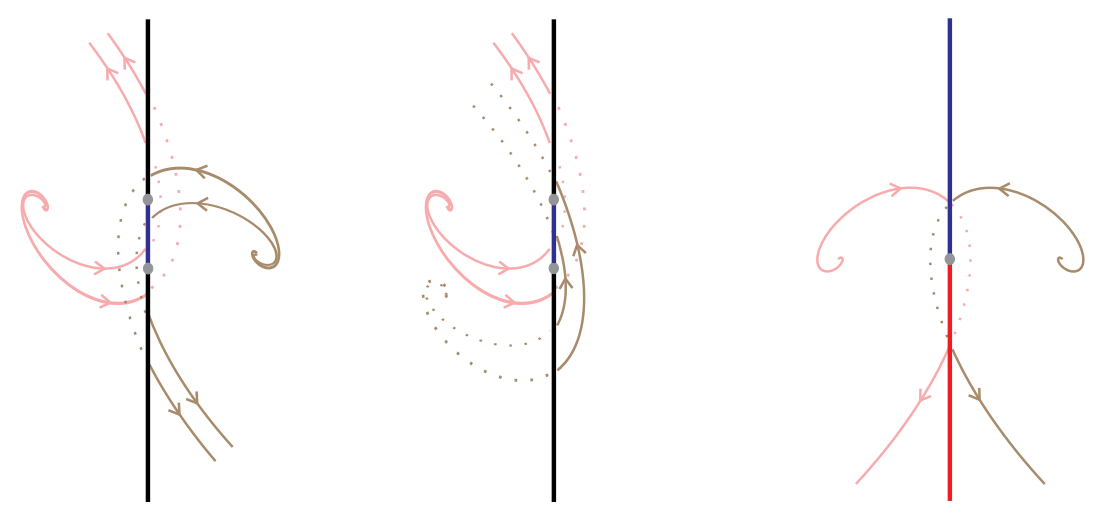

Figure 6: Lines 3, 6 and 10 of Table 3.
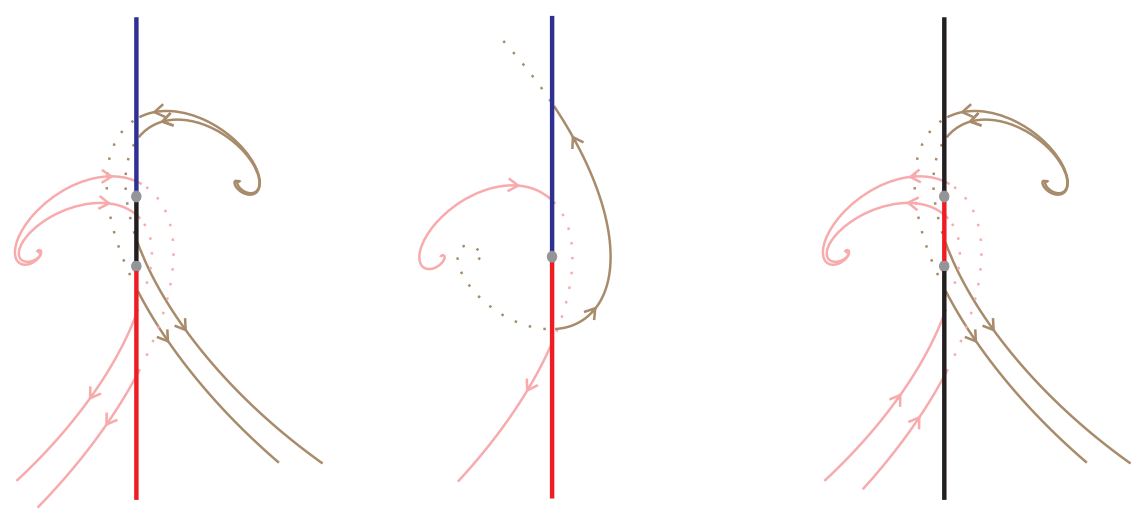

Figure 7: Lines 11, 12 and 17 of Table 3.

$-1,<0,+,<0$, or $+1,<0,-,<0$, or $+1,>0,-,<0$, we have only one possible choice to $s_{1}$. This reduces the number of configurations to 960 . For the other cases with two fold points, for each choice of the parameters $s_{2}, s_{3}$ $s_{4}, s_{5}$ and $s_{6}$ there are two possible choices for $s_{1}$. So we can reduce the number combinations to 160 as desired.

Proof of Theorem 1-(c). These 160 configurations given in Lemma 4, may be reduced to 80 configurations considering the item (i) of relation $R_{3}$ (see Table 3 ). The configurations corresponding to the lines $49,51,50,55,57,56,58,59,60,41$, $42,47,48,45,46,69,70,72,74,73,78,80,61,62,63,64,67,68,52,54,53,43,44,71$, $79,75,76,77,65$ and 66 are related with the first 40 lines of Table 3 by relation $R_{3}$. Moreover, the configurations corresponding to the lines 4, 5, 6, 12, 13, 17, 18, 19, $20,22,25$ and 26 are related with the configurations corresponding to the lines 29 , $30,31,32,33,34,36,37,38,35,39$ and 40 by relation $R_{3}$. 


\begin{tabular}{|c|c|c|c|c|c|c|}
\hline \multicolumn{7}{|c|}{ FOCUS - FOCUS } \\
\hline 1 & $\mathrm{CC}$ & $\overline{\mathbf{R R}}$ & $s_{1}=-1$ & $s_{2}>0$ & $s_{3}=-1$ & $s_{4}>0$ \\
\hline 2 & $\overline{C E C}$ & RR & $s_{1}=-1$ & $s_{2}>0$ & $s_{3}=-1$ & $s_{4}>0$ \\
\hline 3 & $\mathrm{CSC}$ & $\mathbf{R}$ & $s_{1}=-1$ & $s_{2}>0$ & $s_{3}=-1$ & $s_{4}>0$ \\
\hline 4 & $\mathrm{CC}$ & $\overline{\mathbf{R V}}$ & $1=-1$ & $s_{2}>0$ & $3=-1$ & $s_{4}>0$ \\
\hline 5 & CEC & $\mathbf{R V}$ & $s_{1}=-1$ & $s_{2}>0$ & $s_{3}=-1$ & $s_{4}>0$ \\
\hline 6 & $\mathrm{CSC}$ & RV & $s_{1}=-1$ & $s_{2}>0$ & $3=-1$ & $s_{4}>0$ \\
\hline 7 & $\mathrm{CC}$ & $V \mathbf{V}$ & $s_{1}=-1$ & $s_{2}>0$ & $s_{3}=-1$ & $s_{4}>0$ \\
\hline 8 & $\mathrm{CSC}$ & $\mathbf{V V}$ & $s_{1}=-1$ & $s_{2}>0$ & $s_{3}=-1$ & $s_{4}>0$ \\
\hline 9 & CEC & $\overline{\mathrm{VV}}$ & $s_{1}=-1$ & $s_{2}>0$ & $s_{3}=-1$ & $s_{4}>0$ \\
\hline 10 & SE & $\bar{R}$ & $\xi_{1}=+1$ & $s_{2}>0$ & $s_{3}=-1$ & $4>0$ \\
\hline 11 & SCE & $\mathbf{R}$ & $s_{1}=+1$ & $s_{2}>0$ & $s_{3}=-1$ & $s_{4}>0$ \\
\hline 12 & SE & $\mathbf{R V}$ & $s_{1}=+1$ & $s_{2}>0$ & $s_{3}=-1$ & $s_{4}>0$ \\
\hline 13 & SCE & $\mathbf{R V}$ & $s_{1}=+1$ & $s_{2}>0$ & $s_{3}=-1$ & $s_{4}>0$ \\
\hline 14 & SE & $\overline{V V}$ & $s_{1}=+1$ & $s_{2}>0$ & $s_{3}=-1$ & $s_{4}>0$ \\
\hline 15 & SCE & VV & $s_{1}=+1$ & $s_{2}>0$ & $s_{3}=-1$ & $s_{4}>0$ \\
\hline 16 & $\mathrm{CC}$ & $\mathbf{R R}$ & $s_{1}=-1$ & $s_{2}<0$ & $s_{3}=-1$ & $s_{4}>0$ \\
\hline 17 & CEC & $\mathbf{R R}$ & $s_{1}=-1$ & $s_{2}<0$ & $s_{3}=-1$ & $s_{4}>0$ \\
\hline 18 & $\mathrm{CC}$ & $\mathbf{R V}$ & $s_{1}=-1$ & $s_{2}<0$ & $s_{3}=-1$ & $s_{4}>0$ \\
\hline 19 & CSC & $\mathbf{R V}$ & $s_{1}=-1$ & $s_{2}<0$ & $s_{3}=-1$ & $s_{4}>0$ \\
\hline 20 & CEC & RV & $s_{1}=-1$ & $s_{2}<0$ & $s_{3}=-1$ & $s_{4}>0$ \\
\hline 21 & $\mathrm{CC}$ & $\overline{V V}$ & $s_{1}=-1$ & $s_{2}<0$ & $s_{3}=-1$ & $s_{4}>0$ \\
\hline 22 & CSC & VV & $s_{1}=-1$ & $s_{2}<0$ & & \\
\hline 23 & $\overline{\mathrm{SE}}$ & $\overline{R R}$ & $s_{1}=+1$ & $s_{2}<0$ & $s_{3}=-1$ & $s_{4}>0$ \\
\hline 24 & SCE & $R R$ & $1=+1$ & $s_{2}<0$ & $3=-1$ & $4>0$ \\
\hline 25 & $\mathbf{E}$ & RV & $s_{1}=+1$ & $s_{2}<0$ & $s_{3}=-1$ & $4>0$ \\
\hline 26 & SCE & RV & $s_{1}=+1$ & $s_{2}<0$ & $s_{3}=-1$ & $s_{4}>0$ \\
\hline 27 & $\mathrm{SE}$ & $V \mathbf{V}$ & $s_{1}=+1$ & $s_{2}<0$ & $s_{3}=-1$ & $s_{4}>0$ \\
\hline 28 & SCE & $\mathrm{VV}$ & $s_{1}=+1$ & $s_{2}<0$ & $s_{3}=-1$ & $s_{4}>0$ \\
\hline 29 & $C$ & $\overline{\mathbf{R}}$ & $1=-1$ & $s_{2}>0$ & $s_{3}=-1$ & \\
\hline 30 & $\mathrm{EC}$ & $\mathbf{R}$ & $1=-1$ & $s_{2}>0$ & $s_{3}=-1$ & $s_{4}>0$ \\
\hline 31 & CSC & VR & $1=-1$ & $s_{2}>0$ & $s_{3}=-1$ & $4>0$ \\
\hline 32 & E & $\mathbf{R}$ & $1=+1$ & $s_{2}>0$ & $s_{3}=-1$ & $s_{4}>0$ \\
\hline 33 & SCE & $\overline{V R}$ & $s_{1}=+1$ & $s_{2}>0$ & $s_{3}=-1$ & $s_{4}>0$ \\
\hline 34 & $\mathrm{CC}$ & $\mathbf{R}$ & $s_{1}=-1$ & $s_{2}<0$ & $s_{3}=-1$ & $s_{4}>0$ \\
\hline 35 & CEC & $\overline{\mathbf{V V}}$ & $s_{1}=-1$ & $s_{2}<0$ & $s_{3}=-1$ & $s_{4}>0$ \\
\hline 36 & $\overline{\mathrm{C}}$ & $\mathbf{R}$ & $1=-1$ & $s_{2}<0$ & $s_{3}=-1$ & $s_{4}>0$ \\
\hline 37 & $\mathrm{EC}$ & $\overline{J \mathbf{R}}$ & $1=-1$ & $s_{2}<0$ & $s_{3}=-1$ & $s_{4}>0$ \\
\hline 38 & Csc & $\mathbf{R}$ & $1=-1$ & $s_{2}<0$ & $s_{3}=-1$ & $s_{4}>0$ \\
\hline 39 & $\overline{\mathrm{SE}}$ & $\overline{V R}$ & $1=+1$ & $s_{2}<0$ & $s_{3}=-1$ & $s_{4}>0$ \\
\hline 40 & $\overline{C E}$ & VR & $s_{1}=+1$ & $s_{2}<0$ & $s_{3}=-1$ & $s_{4}>0$ \\
\hline 41 & $\overline{\mathbf{S}}$ & $\mathbf{R R}$ & $1=-1$ & $s_{2}>0$ & $s_{3}=+1$ & $s_{4}>0$ \\
\hline 42 & ECS & $\mathbf{R}$ & $s_{1}=-1$ & $s_{2}>0$ & $s_{3}=+1$ & $s_{4}>0$ \\
\hline 43 & $\overline{\mathbf{S}}$ & $\mathbf{R V}$ & $1=-1$ & $s_{2}>0$ & $s_{3}=+1$ & $s_{4}>0$ \\
\hline 44 & $\overline{\mathrm{CS}}$ & $\mathbf{R V}$ & $s_{1}=-1$ & $s_{2}>0$ & $s_{3}=+1$ & $s_{4}>0$ \\
\hline 45 & ES & VV & $s_{1}=-1$ & $s_{2}>0$ & $s_{3}=+1$ & $s_{4}>0$ \\
\hline 46 & $\mathrm{CS}$ & $\overline{J V}$ & $s_{1}=-1$ & $s_{2}>0$ & $s_{3}=+1$ & $s_{4}>0$ \\
\hline 47 & $\bar{S}$ & $\overline{V R}$ & $s_{1}=-1$ & $s_{2}>0$ & $s_{3}=+1$ & $s_{4}>0$ \\
\hline 48 & ECS & $\mathbf{R V}$ & $s_{1}=-1$ & $s_{2}>0$ & $s_{3}=+1$ & $s_{4}>0$ \\
\hline 49 & $\bar{C}$ & R & $1=+1$ & $s_{2}>0$ & $s_{3}=+1$ & $s_{4}>0$ \\
\hline 50 & SC & $\overline{R R}$ & $s_{1}=+1$ & $s_{2}>0$ & $s_{3}=+1$ & $s_{4}>0$ \\
\hline 51 & CEC & RR & $s_{1}=+1$ & $s_{2}>0$ & $s_{3}=+1$ & $s_{4}>0$ \\
\hline 52 & $\mathrm{CC}$ & RV & $s_{1}=+1$ & $s_{2}>0$ & $s_{3}=+1$ & $s_{4}>0$ \\
\hline 53 & CSC & RV & $s_{1}=+1$ & $s_{2}>0$ & $s_{3}=+1$ & $s_{4}>0$ \\
\hline 54 & CEC & RV & $s_{1}=+1$ & $s_{2}>0$ & $s_{3}=+1$ & $s_{4}>0$ \\
\hline 55 & $\mathrm{CC}$ & VR & $s_{1}=+1$ & $s_{2}>0$ & $s_{3}=+1$ & $s_{4}>0$ \\
\hline
\end{tabular}




\begin{tabular}{|l|c|c|c|c|c|c|}
56 & $\mathbf{C S C}$ & $\mathbf{V R}$ & $s_{1}=+1$ & $s_{2}>0$ & $s_{3}=+1$ & $s_{4}>0$ \\
\hline 57 & $\mathbf{C E C}$ & $\mathbf{V R}$ & $s_{1}=+1$ & $s_{2}>0$ & $s_{3}=+1$ & $s_{4}>0$ \\
\hline 58 & $\mathbf{C C}$ & $\mathbf{V V}$ & $s_{1}=+1$ & $s_{2}>0$ & $s_{3}=+1$ & $s_{4}>0$ \\
\hline 59 & $\mathbf{C S C}$ & $\mathbf{V V}$ & $s_{1}=+1$ & $s_{2}>0$ & $s_{3}=+1$ & $s_{4}>0$ \\
\hline 60 & $\mathbf{C E C}$ & $\mathbf{V V}$ & $s_{1}=+1$ & $s_{2}>0$ & $s_{3}=+1$ & $s_{4}>0$ \\
\hline 61 & $\mathbf{E S}$ & $\mathbf{R R}$ & $s_{1}=-1$ & $s_{2}<0$ & $s_{3}=+1$ & $s_{4}>0$ \\
\hline 62 & $\mathbf{E C S}$ & $\mathbf{R R}$ & $s_{1}=-1$ & $s_{2}<0$ & $s_{3}=+1$ & $s_{4}>0$ \\
\hline 63 & $\mathbf{E S}$ & $\mathbf{R V}$ & $s_{1}=-1$ & $s_{2}<0$ & $s_{3}=+1$ & $s_{4}>0$ \\
\hline 64 & $\mathbf{E C S}$ & $\mathbf{R V}$ & $s_{1}=-1$ & $s_{2}<0$ & $s_{3}=+1$ & $s_{4}>0$ \\
\hline 65 & $\mathbf{E S}$ & $\mathbf{V R}$ & $s_{1}=-1$ & $s_{2}<0$ & $s_{3}=+1$ & $s_{4}>0$ \\
\hline 66 & $\mathbf{E C S}$ & $\mathbf{V R}$ & $s_{1}=-1$ & $s_{2}<0$ & $s_{3}=+1$ & $s_{4}>0$ \\
\hline 67 & $\mathbf{E S}$ & $\mathbf{V V}$ & $s_{1}=-1$ & $s_{2}<0$ & $s_{3}=+1$ & $s_{4}>0$ \\
\hline 68 & $\mathbf{E C S}$ & $\mathbf{V V}$ & $s_{1}=-1$ & $s_{2}<0$ & $s_{3}=+1$ & $s_{4}>0$ \\
\hline 69 & $\mathbf{C C}$ & $\mathbf{R R}$ & $s_{1}=+1$ & $s_{2}<0$ & $s_{3}=+1$ & $s_{4}>0$ \\
\hline 70 & $\mathbf{C S C}$ & $\mathbf{R R}$ & $s_{1}=+1$ & $s_{2}<0$ & $s_{3}=+1$ & $s_{4}>0$ \\
\hline 71 & $\mathbf{C E C}$ & $\mathbf{R R}$ & $s_{1}=+1$ & $s_{2}<0$ & $s_{3}=+1$ & $s_{4}>0$ \\
\hline 72 & $\mathbf{C C}$ & $\mathbf{R V}$ & $s_{1}=+1$ & $s_{2}<0$ & $s_{3}=+1$ & $s_{4}>0$ \\
\hline 73 & $\mathbf{C S C}$ & $\mathbf{R V}$ & $s_{1}=+1$ & $s_{2}<0$ & $s_{3}=+1$ & $s_{4}>0$ \\
\hline 74 & $\mathbf{C E C}$ & $\mathbf{R V}$ & $s_{1}=+1$ & $s_{2}<0$ & $s_{3}=+1$ & $s_{4}>0$ \\
\hline 75 & $\mathbf{C C}$ & $\mathbf{V R}$ & $s_{1}=+1$ & $s_{2}<0$ & $s_{3}=+1$ & $s_{4}>0$ \\
\hline 76 & $\mathbf{C S C}$ & $\mathbf{V R}$ & $s_{1}=+1$ & $s_{2}<0$ & $s_{3}=+1$ & $s_{4}>0$ \\
\hline 77 & $\mathbf{C E C}$ & $\mathbf{V R}$ & $s_{1}=+1$ & $s_{2}<0$ & $s_{3}=+1$ & $s_{4}>0$ \\
\hline 78 & $\mathbf{C C}$ & $\mathbf{V V}$ & $s_{1}=+1$ & $s_{2}<0$ & $s_{3}=+1$ & $s_{4}>0$ \\
\hline 79 & $\mathbf{C S C}$ & $\mathbf{V V}$ & $s_{1}=+1$ & $s_{2}<0$ & $s_{3}=+1$ & $s_{4}>0$ \\
\hline 80 & $\mathbf{C E C}$ & $\mathbf{V V}$ & $s_{1}=+1$ & $s_{2}<0$ & $s_{3}=+1$ & $s_{4}>0$ \\
\hline & & & & & \\
\hline
\end{tabular}

Table 3: Possible configurations for the focus-focus case using the item (i) of relation $R_{3}$. The lines $1-28$ represent the configurations of Theorem 1-(c). The highlighted lines present the necessary configurations to the existence of closed sliding poly-trajectories.
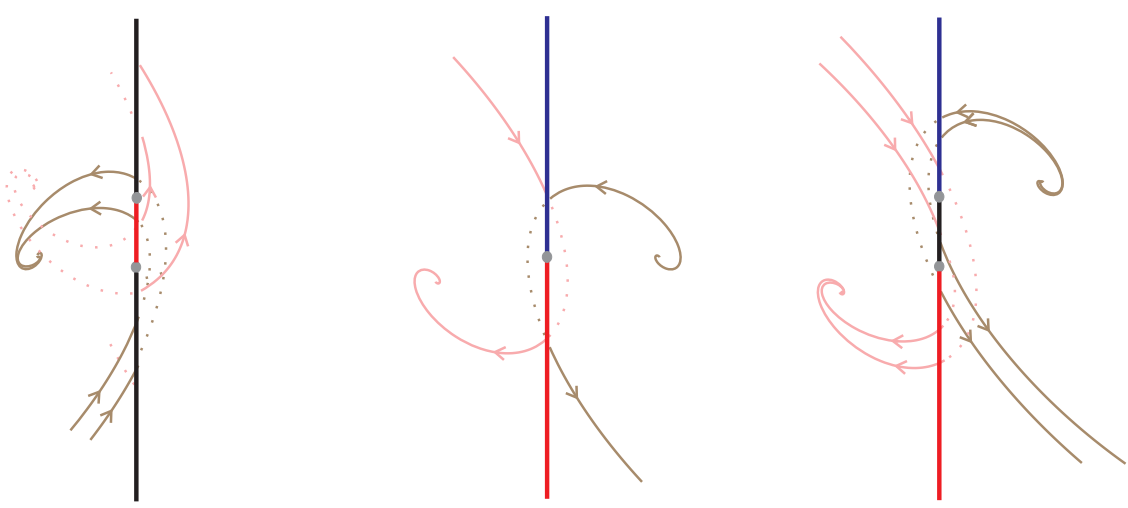

Figure 8: Lines 20, 23 and 24 of Table 3. 

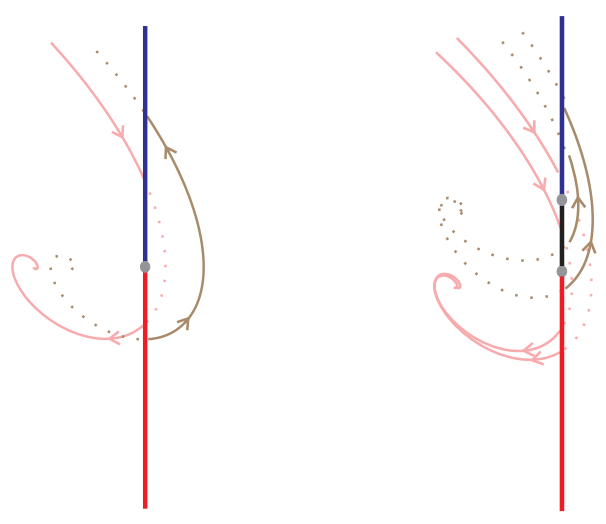

Figure 9: Lines 25 and 26 of Table 3.

\subsection{Proof of Theorem 2}

Let $F$ be a vector field given by (1), $p_{0}$ and $p_{1}$, be the equilibrium points and, $\varphi$ and $\psi$ be the flows of $F^{-}$and $F^{+}$respectively. Suppose that $\mathbf{W}_{L}^{s}=G_{1}^{-1}(\{0\})$, $\mathbf{W}_{L}^{u}=G_{2}^{-1}(\{0\}), \mathbf{W}_{R}^{s}=G_{3}^{-1}(\{0\})$ and $\mathbf{W}_{R}^{u}=G_{4}^{-1}(\{0\})$, where $G_{i}: \mathbb{R}^{2} \rightarrow \mathbb{R}$, $i=1, . .4$, are smooth functions and 0 is a regular value of $G_{i}$.

Consider the following lemma.

Lemma 5. PWL vector fields with configurations given on lines 1, 3, 4, 6, 8-10, 12, 16, 17, 19-22 of Table 1 do not have closed sliding poly-trajectories.

Proof. The vector fields corresponding to configurations given on lines 1, 3, 4, 6, $8-10,12,16,17,19-22$ of Table 1 do not have closed sliding poly-trajectories because they satisfy the following sentences, which provide the impossibility of a periodic motion, more specifically:

- For lines 1,8 and $10, i=3$ or 4 we have

$$
\begin{gathered}
\lim _{t \rightarrow+\infty} G_{i-2}(\varphi( \pm t, p))=0 \text { or } \lim _{t \rightarrow+\infty} \varphi( \pm t, p) \rightarrow p_{0} \text {, and } \\
\lim _{t \rightarrow+\infty} G_{i}(\psi( \pm t, p))=0 \text { or } \lim _{t \rightarrow+\infty} \psi( \pm t, p) \rightarrow p_{1}, p \in \Sigma^{S} \cup \Sigma^{e} .
\end{gathered}
$$

- For lines 4, 6, 17, 19 and 21 we have

$$
\begin{gathered}
\lim _{t \rightarrow+\infty} G_{1}(\varphi(-t, p))=0 \text { or } \lim _{t \rightarrow+\infty} G_{2}(\varphi(t, p))=0 \text {, and } \\
\lim _{t \rightarrow+\infty} G_{3}(\psi(-t, p))=0 \text { or } \lim _{t \rightarrow+\infty} G_{4}(\psi(t, p))=0, p \in \Sigma^{s} \cup \Sigma^{e} .
\end{gathered}
$$

- For lines $3,9, i=3$ or 4 , we have

$$
\begin{gathered}
\lim _{t \rightarrow+\infty} G_{i-2}(\varphi( \pm t, p))=0 \text { or } \lim _{t \rightarrow+\infty} \varphi( \pm t, p)=p_{0} \text {, and } \\
\lim _{t \rightarrow+\infty} G_{i}(\psi( \pm t, p))=0 \text { or } \lim _{t \rightarrow+\infty} \psi( \pm t, p)=p_{1} \text { or } \\
\psi\left( \pm t_{1}, p\right) \in \Sigma^{c}, p \in \Sigma^{S} \cup \Sigma^{e}, t_{1}>0 .
\end{gathered}
$$


- For line 12 we have

$$
\begin{gathered}
\lim _{t \rightarrow+\infty} G_{2}(\varphi(t, p))=0 \text { or } \lim _{t \rightarrow+\infty} \varphi(t, p)=p_{0} \text { or, } \varphi\left(t_{1}, p\right) \in \Sigma^{c} \text { and } \\
\psi\left(t_{2}, \varphi\left(t_{1}, p\right)\right) \in \Sigma^{c} \text { or } \lim _{t \rightarrow+\infty} G_{4}(\psi(t, \varphi(t, p)))=0, t_{1}, t_{2}>0, p \in \Sigma^{e} . \\
\lim _{t \rightarrow+\infty} G_{4}(\psi(t, p))=0 \text { or } \lim _{t \rightarrow+\infty} \psi(t, p)=p_{1} \text { or, } \psi\left(s_{1}, p\right) \in \Sigma^{c} \text { and } \\
\varphi\left(s_{2}, \psi\left(s_{1}, p\right)\right) \in \Sigma^{c} \text { or } \lim _{t \rightarrow+\infty} G_{2}(\varphi(t, \psi(t, p)))=0, s_{1}, s_{2}>0 p \in \Sigma^{e} .
\end{gathered}
$$

- For line 16 we have

$$
\begin{array}{r}
\lim _{t \rightarrow+\infty} G_{4}(\psi(t, p))=0 \text { or } \lim _{t \rightarrow+\infty} G_{3}(\psi(-t, p))=0, p \in \Sigma \text {, and } \\
\lim _{t \rightarrow+\infty} G_{2}(\varphi(t, p))=0 \text { or } \varphi\left(t_{1}, p\right) \in \Sigma^{c} \text { or } \lim _{t \rightarrow+\infty} \varphi(t, p)=p_{0}, \\
p \in \Sigma^{e}, t_{1}>0 .
\end{array}
$$

- For line 20 we have

$$
\begin{aligned}
& \lim _{t \rightarrow+\infty} G_{4}(\psi(t, p))=0 \text { or } \lim _{t \rightarrow+\infty} \psi(t, p)=p_{1} \text { or } \psi\left(t_{1}, p\right) \in \Sigma^{S} \text { or, } \\
& \psi\left(t_{2}, p\right) \in \Sigma^{c} \text { and } \varphi\left(t_{3}, \psi\left(t_{2}, p\right)\right) \in \Sigma^{s}, p \in \Sigma^{e}, t_{1}, t_{2}, t_{3}>0 \text {. } \\
& \lim _{t \rightarrow+\infty} G_{2}(\varphi(t, p))=0 \text { or } \lim _{t \rightarrow+\infty} \varphi(t, p)=p_{0} \text { or } \varphi\left(s_{1}, p\right) \in \Sigma^{s} \text {, } \\
& p \in \Sigma^{e}, s_{1}>0 \text {. }
\end{aligned}
$$

- For line 22 we have

$$
\begin{gathered}
\lim _{t \rightarrow+\infty} G_{4}(\psi(t, p))=0 \text { or } \lim _{t \rightarrow+\infty} G_{3}(\psi(-t, p))=0, p \in \Sigma, \text { and } \\
\varphi\left(t_{1}, p\right) \in \Sigma^{c} \text { or } \varphi\left(t_{1}, p\right) \in \Sigma^{S} \text { or } \lim _{t \rightarrow+\infty} G_{2}(\varphi(t, p))=0 \text { or } \\
\lim _{t \rightarrow+\infty} \varphi(t, p)=p_{0}, p \in \Sigma^{e}, t_{1}>0 .
\end{gathered}
$$

The times $t_{i}, s_{i}$, given in (5)-(11) are the first return times on $\Sigma$ for $\varphi$ or $\psi$.

Lemma 6. A PWL vector field with configuration given on line 14 of Table 1, does not have closed sliding poly-trajectories.

Proof. If there exists a closed sliding poly-trajectory, then it is given by Figure 10 $((\mathrm{A})$ or $(\mathrm{B}))$. Without loss of generality we suppose that the fold point of $F^{-}$and $F^{+}$is $(0,0)$. So, $b_{1}^{-}=b_{1}^{+}=0$. We have

$$
a_{11}^{-} a_{22}^{-}-a_{12}^{-} a_{21}^{-}<0, \quad a_{11}^{+} a_{22}^{+}-a_{12}^{+} a_{21}^{+}<0 .
$$

The equilibrium points of $F^{-}$and $F^{+}$are given respectively by

$$
x^{\mp}=\frac{a_{12}^{\mp} b_{2}^{\mp}}{a_{11}^{\mp} a_{22}^{\mp}-a_{21}^{\mp} a_{12}^{\mp}}, \quad y^{\mp} \frac{a_{11}^{\mp} b_{2}^{\mp}}{a_{11}^{\mp} a_{22}^{\mp}-a_{21}^{\mp} a_{12}^{\mp}}
$$


and satisfy $x^{-}<0, \quad x^{+}>0$. We have that $a_{12}^{+} y<0, a_{12}^{-} y>0, y<0$. Thus, $a_{12}^{-}<0, a_{12}^{+}>0$ and $b_{2}^{-}, b_{2}^{+}<0$. The sliding vector field is given by

$$
\dot{x}=0, \quad g(y)=\frac{\left(a_{12}^{+} a_{22}^{-}-a_{12}^{-} a_{22}^{+}\right) y+a_{12}^{+} b_{2}^{-}-a_{12}^{-} b_{2}^{+}}{a_{12}^{+}-a_{12}^{-}}, \quad y \neq 0,
$$

and since $a_{12}^{+} a_{22}^{-}-a_{12}^{-} a_{22}^{+} \neq 0$, it has an equilibrium point given by $y_{0}=\frac{a_{12}^{-} b_{2}^{+}-a_{12}^{+} b_{2}^{-}}{a_{12}^{+} a_{22}^{-}-a_{12}^{-} a_{22}^{+}}$. The derivation of $g(y)$ is given by $g^{\prime}(y)=\frac{a_{12}^{+} a_{22}^{-}-a_{12}^{-} a_{22}^{+}}{a_{12}^{+}-a_{12}^{-}}$.

(i) If $a_{12}^{+} a_{22}^{-}-a_{12}^{-} a_{22}^{+}>0($ resp. $<0)$, then $y_{0}>0($ resp. $<0)$ and $g^{\prime}(y)>0$ $($ resp. $<0)$.

(ii) If $a_{12}^{+} a_{22}^{-}-a_{12}^{-} a_{22}^{+}=0$, then $g(y)<0$ for all $y \neq 0$.

So the flow on $\Sigma$ is given in Figure $10((C),(D)$ and $(E))$. In any case it is impossible the existence of a closed sliding poly-trajectory. In fact, consider an orbit of $F^{\mp}$ with the orientation given in Figure $10((\mathrm{~A})$ or $(\mathrm{B}))$, connecting $y_{1}, y_{2} \in \Sigma$, $y_{1} y_{2}<0$ and observe that the orientation is not compatible with the cases on Figure $10((\mathrm{C}),(\mathrm{D})$ and $(\mathrm{E}))$.

Proof of Theorem 2-(a). The proof of item (a) is an immediate consequence of Lemmas 5 and 6.

Lemma 7. PWL vector fields with configurations given on lines 2-4, 5, 7-10, 12-16, 17-20, 22, 24, and 28 of Table 2, do not have closed sliding poly-trajectories.

Proof. The proof is similar to Lemma 5.

Lemma 8. A PWL vector field with configuration given on line 23 of Table 2, does not have closed sliding poly-trajectories.

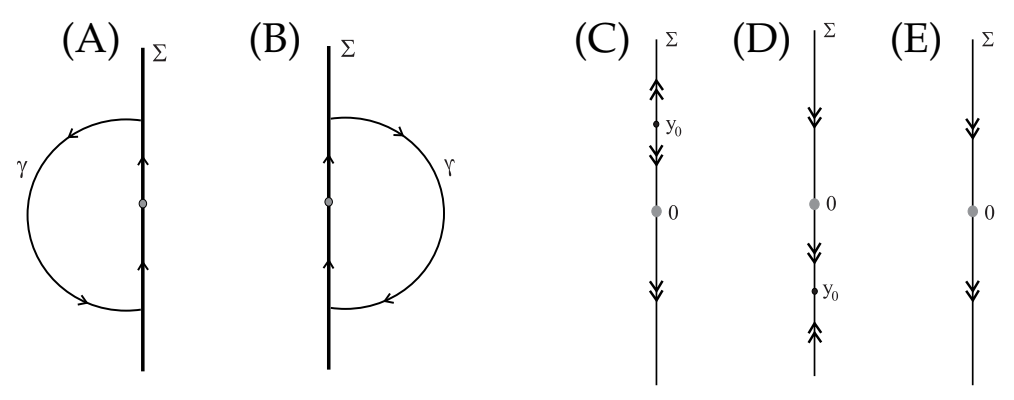

Figure 10: The figures (A) and (B) represent the possible closed sliding poly-trajectories given by a PWL vector field with configuration given on line 14 of Table 1 . The figures (C), (D) and (E) represent the possible flows on $\Sigma$ for $a_{12}^{+} a_{22}^{-}-a_{12}^{-} a_{22}^{+}>0, a_{12}^{+} a_{22}^{-}-a_{12}^{-} a_{22}^{+}<0$ and $a_{12}^{+} a_{22}^{-}-a_{12}^{-} a_{22}^{+}=0$, respectively. 
Proof. If there is a closed sliding poly-trajectory, then it is given in Figure 11. As in the Lemma 6, we have $b_{1}^{-}=b_{1}^{+}=0, a_{12}^{-}>0, a_{12}^{+}<0, b_{2}^{-}>0, b_{2}^{+}>0$, and the sliding vector field is given in (12).

(i) If $a_{12}^{+} a_{22}^{-}-a_{12}^{-} a_{22}^{+}>0$, then $y_{0}>0$ and $g^{\prime}(y)<0$.

(ii) If $a_{12}^{+} a_{22}^{-}-a_{12}^{-} a_{22}^{+}<0$, then $y_{0}<0$ and $g^{\prime}(y)>0$.

(iii) If $a_{12}^{+} a_{22}^{-}-a_{12}^{-} a_{22}^{+}=0$, then $g(y)>0$ for all $y \neq 0$.

The flow on $\Sigma$ is given in Figure $11((\mathrm{C}),(\mathrm{D})$ and $(\mathrm{E}))$. In any case it is impossible the existence of a closed sliding poly-trajectory because the orientation on $\Sigma$ is not compatible with the orientation in Figure 11 ((A) and (B)).

Proof of Theorem 2-(b). The proof of item (b) is an immediate consequence of Lemmas 7 and 8.

Lemma 9. PWL vector fields with configurations given on lines $2,3,5,8,9,13,15,19$, 22 and 28 of Table 3, do not have closed sliding poly-trajectories.

Proof. The proof is similar to Lemma 5.

Lemma 10. A PWL vector field with configuration given on line 14 or 27 of Table 3, does not have closed sliding poly-trajectories.

Proof. The proof is analogous to the Lemma 8.

Proof of Theorem 2-(c). The proof of item (c) is an immediate consequence of Lemmas 9 and 10.

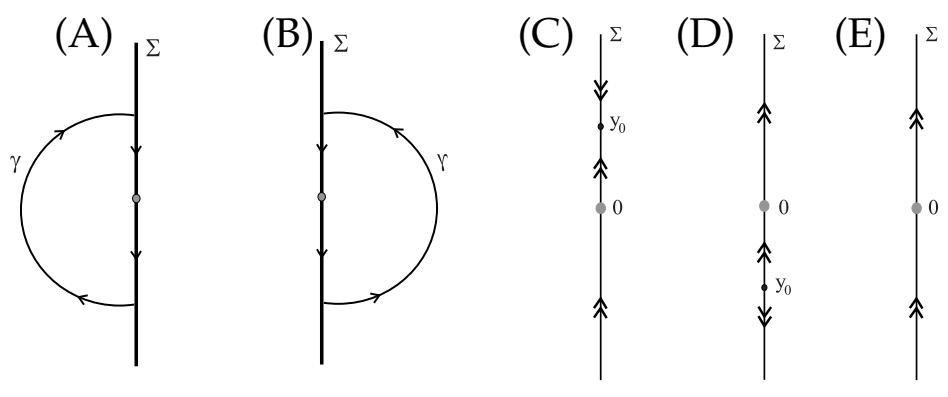

Figure 11: The figures (A) and (B) represent the possible closed sliding poly-trajectories for a PWL vector field with configuration given on line 23 of Table 2. The figures (C), (D) and (E) represent the flows on $\Sigma$ for a PWL vector field with configuration given on line 23 of Table 2, for items (i), (ii) and (iii), respectively. 


\section{Proof of Theorem 3.}

In this section we proof the Theorem 3. We obtain conditions for the PWL vector fields corresponding to line 13 of Table 1, lines 1, 6, 11, 17, 21, $25-27$ of Table 2 and lines $3,6,10-12,20,23-26$ of Table 3 have or not closed sliding polytrajectories.

Proposition 1. Consider a PWL vetor field with configuration given on line 13 of table 1.

(a) If $a_{12}^{-} b_{2}^{+}-a_{12}^{+} b_{2}^{-}<0$, then it has a family of closed sliding poly-trajectories.

(b) If $a_{12}^{-} b_{2}^{+}-a_{12}^{+} b_{2}^{-} \geq 0$, then it does not have closed sliding poly-trajectories.

Proof. If there is any closed sliding poly-trajectory, then it is like the one given on Figure $10(\mathrm{~A})$. As in the proof of Lemma 6, we have $b_{1}^{-}=b_{1}^{+}=0, a_{12}^{-}<0, a_{12}^{+}>0$, $b_{2}^{-}<0, b_{2}^{+}>0$, and the sliding vector field, $y_{0}$ and $g^{\prime}(y)$ are the same.

Suppose that $a_{12}^{-} b_{2}^{+}-a_{12}^{+} b_{2}^{-}<0$ (resp. $>0$ ).

(i) (resp. iv) If $a_{12}^{+} a_{22}^{-}-a_{12}^{-} a_{22}^{+}<0$, then $y_{0}>0$ (resp. $<0$ ) and $g^{\prime}(y)<0$.

(ii) (resp. v) If $a_{12}^{+} a_{22}^{-}-a_{12}^{-} a_{22}^{+}>0$, then $y_{0}<0$ (resp. $>0$ ) and $g^{\prime}(y)>0$.

(iii) (resp. vi) If $a_{12}^{+} a_{22}^{-}-a_{12}^{-} a_{22}^{+}=0$, then $g(y)>0$ (resp. $<0$ ) for all $y \neq 0$.

Suppose that $a_{12}^{-} b_{2}^{+}-a_{12}^{+} b_{2}^{-}=0$.

(vii) (resp. viii) If $a_{12}^{+} a_{22}^{-}-a_{12}^{-} a_{22}^{+}<0$ (resp. $>0$ ), then $g(y)<0$ (resp. $>0$ ) for $y>0$ and $g(y)>0$ (resp. $<0)$ for $y<0$.

If $a_{12}^{+} a_{22}^{-}-a_{12}^{-} a_{22}^{+}=a_{12}^{-} b_{2}^{+}-a_{12}^{+} b_{2}^{-}=0$, then the sliding vector field is identically zero and this implies the non-existence of closed sliding poly-trajectories. It is possible the existence of a family of closed sliding poly-trajectories for item (i) (resp. (ii)), since the arcs of $F^{-}$meet $\Sigma$ in points less (resp. greater) than $y_{0}$. Obviously, for item (iii) always it is possible the existence of a family of closed sliding poly-trajectories because the orientation on $\Sigma$ is the same given in Figure 10 (A). For items (iv), (v), (vi), (vii) and (viii) it is not possible the existence of closed sliding poly-trajectories because the orientation on $\Sigma$ is not compatible with the one given in Figure 10 (A). Note that we obtain a family of closed sliding poly-trajectories instead of isolated closed sliding poly-trajectories because they do not satisfy item (3) of Definition 5.

Similar arguments are used to proof the following Propositions $2-20$.

Proposition 2. Consider a PWL vetor field with configuration given on line 1 of Table 2.

(a) If $a_{12}^{+} b_{2}^{-}-a_{22}^{+} b_{1}^{-} \geq 0$, then it has a hyperbolic closed sliding poly-trajectory.

(b) If $a_{12}^{+} b_{2}^{-}-a_{22}^{+} b_{1}^{-}<0$, then it can have or not a hyperbolic closed sliding polytrajectory.

Proposition 3. A PWL vector field with configuration given on line 6 of Table 2 can have or not a hyperbolic closed sliding poly-trajectory. 


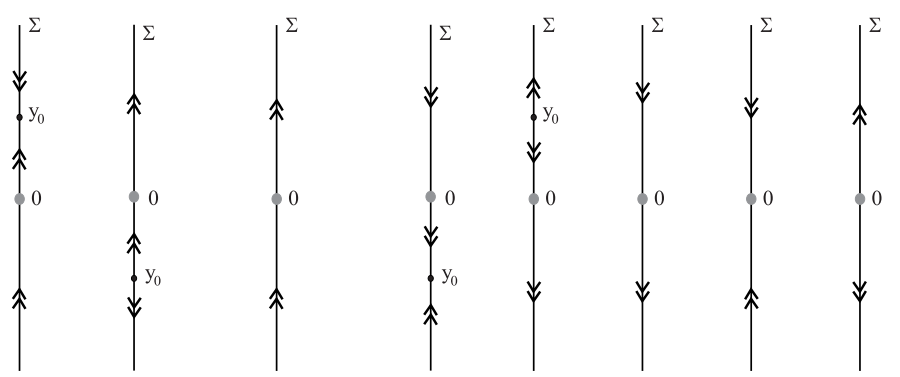

Figure 12: Cases corresponding to items (i), (ii), (iii), (iv), (v), (vi), (vii) and (viii), respectively.

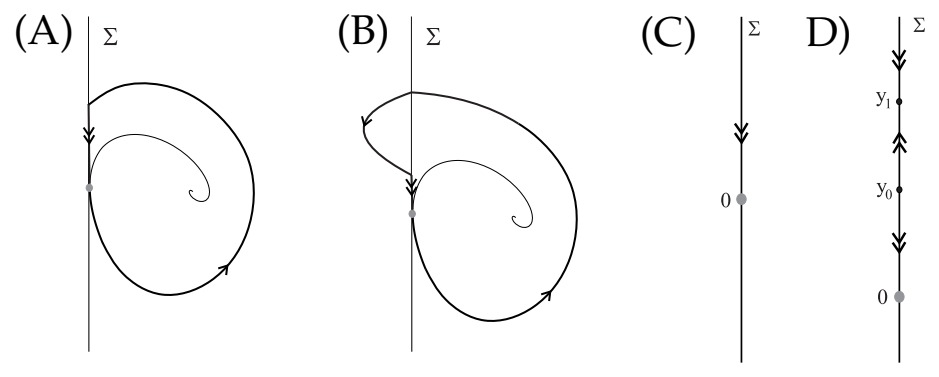

Figure 13: The figures (A), (C) and (D) present the possible closed sliding poly-trajectory for a PWL vector field with configuration given on line 1 of Table 2 and the flows on $\Sigma$ corresponding to items (a) and (b), respectively. The figures (A) and (B) also represent the possible closed sliding poly-trajectories for a PWL vector field with configuration given on line 11 of Table 2.

If there is a closed sliding poly-trajectory, then it is given on Figure 13 (A).

Proposition 4. Consider a PWL vector field with configuration given on line 11 of Table 2. Let $a=a_{12}^{+} a_{22}^{-}-a_{12}^{-} a_{22}$ and $b=a_{12}^{+} b_{2}^{-}-a_{12}^{-} b_{2}^{+}-a_{22}^{+} b_{1}^{-}$. If $a \leq 0$ or $b \geq 0$, then it can have or not a hyperbolic closed sliding poly-trajectory.

Proposition 5. Consider a PWL vector field with configuration given on line 17 of Table 2. Thus it can have or not a hyperbolic closed sliding poly-trajectory.

If there is a closed sliding poly-trajectory, then it is given in Figure 13 (A).

Proposition 6. Consider a PWL vector field with configuration given on line 21 of Table 2.

(a) If $a_{12}^{-} b_{2}^{+}-a_{12}^{+} b_{2}^{-}<0$ and $a_{12}^{+} a_{22}^{-}-a_{12}^{-} a_{22}^{+} \leq 0$, then it has two families of closed sliding poly-trajectories and a hyperbolic closed sliding poly-trajectory.

(b) If $a_{12}^{-} b_{2}^{+}-a_{12}^{+} b_{2}^{-}<0$ and $a_{12}^{+} a_{22}^{-}-a_{12}^{-} a_{22}^{+}>0$, then it has two families of closed sliding poly-trajectories and it can have more one family of closed sliding polytrajectories and a hyperbolic closed sliding poly-trajectory.

(c) If $a_{12}^{-} b_{2}^{+}-a_{12}^{+} b_{2}^{-}=0$ and $a_{12}^{+} a_{22}^{-}-a_{12}^{-} a_{22}^{+}<0$, then it has only a hyperbolic closed sliding poly-trajectory. 


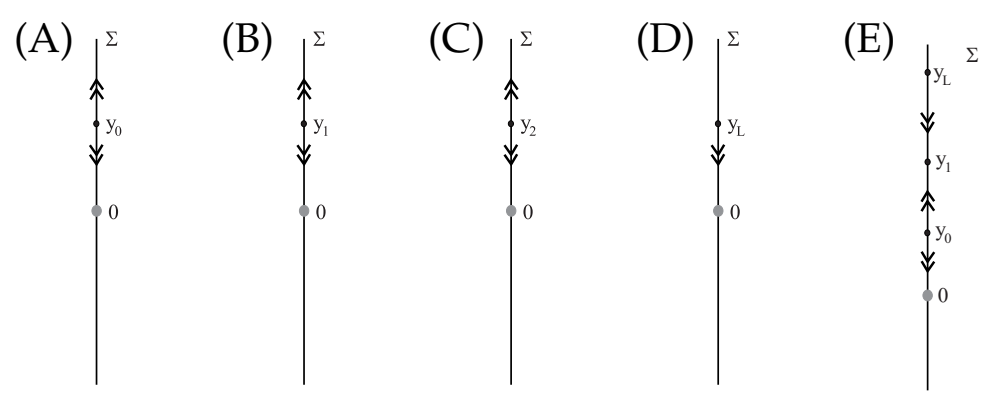

Figure 14: The figures (A), (B) and (C) represent the flows on $\Sigma$ for a PWL vector field with configuration given on line 6 of Table 2, for $a_{22}^{+} b_{1}^{-}-a_{12}^{+} b_{2}^{-}>0, a_{22}^{+} b_{1}^{-}-a_{12}^{+} b_{2}^{-}<0$ and $a_{22}^{+} b_{1}^{-}-a_{12}^{+} b_{2}^{-}=0$, respectively. The figures (D) and (E) represent the flows on $\Sigma$ for a PWL vector field with configuration given on lines 11 and 17 of Table 2.

(d) If $a_{12}^{-} b_{2}^{+}-a_{12}^{+} b_{2}^{-}>0$ and $a_{12}^{+} a_{22}^{-}-a_{12}^{-} a_{22}^{+}<0$, or $a_{12}^{-} b_{2}^{+}-a_{12}^{+} b_{2}^{-}>0$ and $a_{12}^{+} a_{22}^{-}-a_{12}^{-} a_{22}^{+}>0$, or $a_{12}^{-} b_{2}^{+}-a_{12}^{+} b_{2}^{-}>0$ and $a_{12}^{+} a_{22}^{-}-a_{12}^{-} a_{22}^{+}=0$, or $a_{12}^{-} b_{2}^{+}-$ $a_{12}^{+} b_{2}^{-}=0$ and $a_{12}^{+} a_{22}^{-}-a_{12}^{-} a_{22}^{+}>0$, then it does not have closed sliding polytrajectories.

If there is a closed sliding poly-trajectory, then it is given in Figure 15 and the flows on $\Sigma$ are given in Figure 12 for each item of Proposition 1.

Proposition 7. Consider a PWL vector field with configuration given on line 25 of Table 2.

(a) If $a_{12}^{+} a_{22}^{-}-a_{12}^{-} a_{22}^{+} \geq 0$, then it has a family of closed sliding poly-trajectories and $a$ hyperbolic poly-trajectory.

(b) If $a_{12}^{+} a_{22}^{-}-a_{12}^{-} a_{22}^{+}<0$, then it can have a family of closed sliding poly-trajectories and a hyperbolic poly-trajectory.

Proposition 8. Consider a PWL vector field with configuration given on line 26 of Table 2. Let $a=a_{12}^{+} a_{22}^{-}-a_{12}^{-} a_{22}$ and $b=a_{12}^{+} b_{2}^{-}-a_{12}^{-} b_{2}^{+}-a_{22}^{+} b_{1}^{-}$.

(a) If $a<0$ or, $a \geq 0$ and $b<0$, then it can have or not a hyperbolic closed sliding poly-trajectory.

(b) For all other signals of $a$ and $b$, it has a hyperbolic closed sliding poly-trajectory.

If there is a closed sliding poly-trajectory, then it is given on Figure 13 (A).

Proposition 9. Consider a PWL vector field with configuration given on line 27 of Table 2.

(a) If $a_{12}^{-} b_{2}^{+}-a_{12}^{+} b_{2}^{-}<0$, then it has a family of closed sliding poly-trajectories.

(b) If $a_{12}^{-} b_{2}^{+}-a_{12}^{+} b_{2}^{-} \geq 0$, then it does not have closed sliding poly-trajectories.

If there is any closed sliding poly-trajectory, then it is like given on Figure 11 (B). 


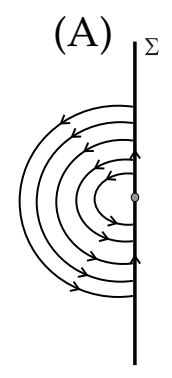

(B)

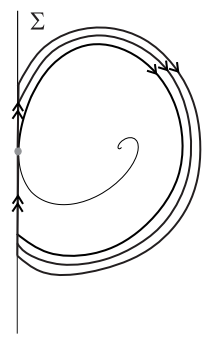

(C)

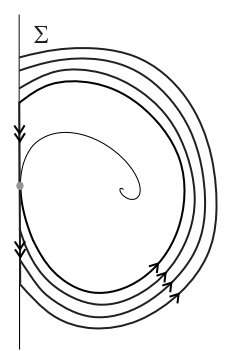

Figure 15: The figures (A) and (B) represent the possible closed sliding polytrajectories for a PWL vector field with configuration given on line 21 of Table 2 and figure $(\mathrm{C})$ represent the possible closed sliding poly-trajectories for a PWL vector field with configuration given on line 25 of Table 2.

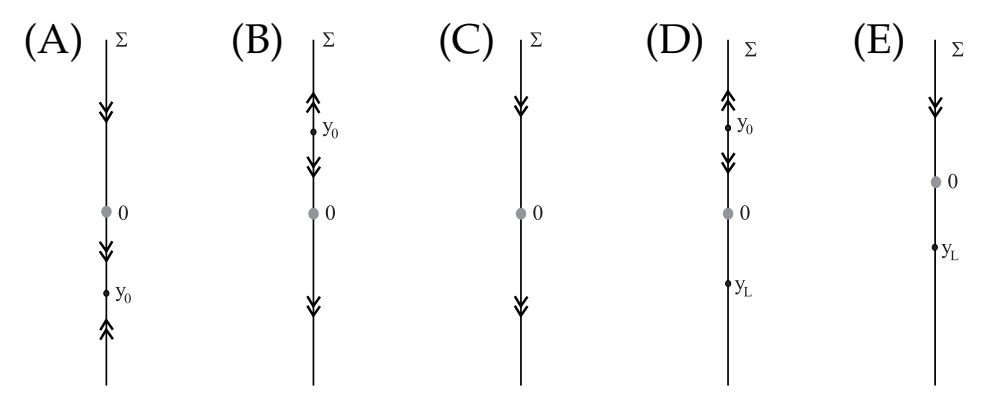

Figure 16: The figures (A), (B) and (C) represent the flows on $\Sigma$ for a PWL vector field given on line 25 of Table 2 for $a_{12}^{+} a_{22}^{-}-a_{12}^{-} a_{22}^{+}>0, a_{12}^{+} a_{22}^{-}-a_{12}^{-} a_{22}^{+}<0$ and $a_{12}^{+} a_{22}^{-}-a_{12}^{-} a_{22}^{+}=$ 0 , respectively. The figures (D) and (E) represent the flows on $\Sigma$ for line 26 of Table 2 for $a<0$ and, $a \geq 0$ and $b>0$, respectively.

Proposition 10. Consider a PWL vector field with configuration given on line 3 of Table 3. Let $a=a_{12}^{+} a_{22}^{-}-a_{12}^{-} a_{22}^{+}$and $b=a_{12}^{+} b_{2}^{-}-a_{12}^{-} b_{2}^{+}-a_{22}^{+} b_{1}^{-}$.

(a) If $a<0$ or, $a \geq 0$ and $b<0$, then it can have one or two hyperbolic closed sliding poly-trajectories.

(b) For all other signals of $a$ and $b$, only $F^{+}$can have a hyperbolic closed sliding polytrajectory.

The flow on $\Sigma$ is given in Figure $14((\mathrm{E}))$.

Proposition 11. Consider a PWL vector field with configuration given on line 6 of Table 3. Let $a=a_{12}^{+} a_{22}^{-}-a_{12}^{-} a_{22}$ and $b=a_{12}^{+} b_{2}^{-}-a_{12}^{-} b_{2}^{+}-a_{22}^{+} b_{1}^{-}$. If $a \geq 0$ or $b \geq 0$, then it can have a hyperbolic closed sliding poly-trajectory.

Proposition 12. Consider a PWL vetor field with configuration given on line 10 of Table 3.

(a) If $a_{12}^{+} a_{22}^{-}-a_{12}^{-} a_{22}^{+} \geq 0$, then it has two families of closed sliding poly-trajectories and two hyperbolic closed sliding poly-trajectories. 


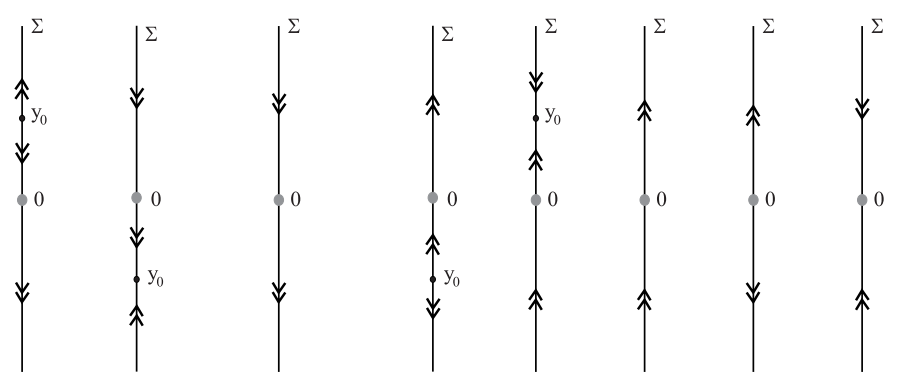

Figure 17: Flows on $\Sigma$ for a PWL with configuration given on line 27 of Table 2. The three first figures correspond to item (a) and the last five figures correspond to item (b).
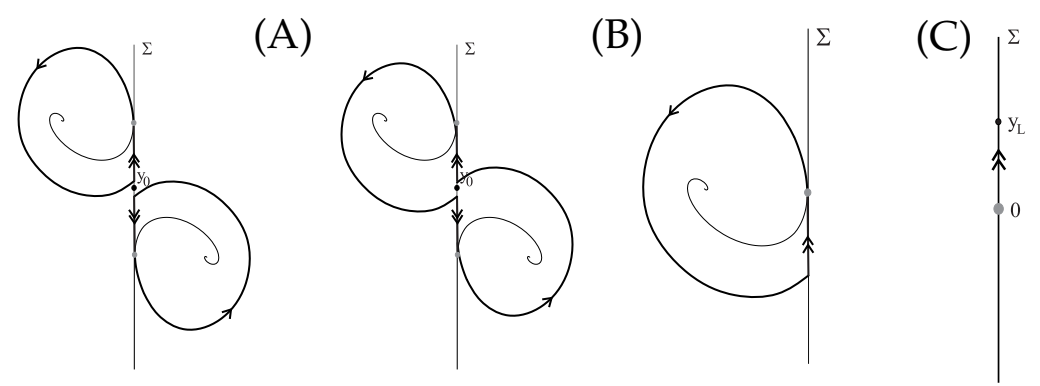

Figure 18: The figures (A) and (B) represent the possible closed sliding poly-trajectories for a PWL vector field with configuration given on lines 3 and 6 of Table 3 . The figure (C) represent the flow on $\Sigma$ for a PWL vector field with configuration given on line 6 of Table 3.

(b) If $a_{12}^{+} a_{22}^{-}-a_{12}^{-} a_{22}^{+}<0$, then it can have one or two families of closed sliding polytrajectories and one or two hyperbolic closed sliding poly-trajectories.

Proposition 13. Consider a PWL vector field with configuration given on line 11 of Table 3. Let $a=a_{12}^{+} a_{22}^{-}-a_{12}^{-} a_{22}$ and $b=a_{12}^{+} b_{2}^{-}-a_{12}^{-} b_{2}^{+}-a_{22}^{+} b_{1}^{-}$.

(a) If $a \geq 0$ and $b \geq 0$, then it has a hyperbolic closed sliding poly-trajectory.

(b) For any other signals of $a$ and $b$, it can have a hyperbolic closed sliding polytrajectory.

If there is a closed sliding poly-trajectory, then it is given on Figure 13 (A). The flow on $\Sigma$ corresponding to item (a) is given in Figure 13 (C) and one corresponding to item (b) is given in Figure 13 (D) and Figure 14 (A).

Proposition 14. Consider a PWL vector field with configuration given on line 12 of Table 3.

(a) If $a_{12}^{-} b_{2}^{+}-a_{12}^{+} b_{2}^{-}<0$ and $a_{12}^{+} a_{22}^{-}-a_{12}^{-} a_{22}^{+}<0$, then it has a family of closed sliding poly-trajectories and it can have more one family of closed sliding poly-trajectories and a hyperbolic closed sliding poly-trajectory.

(b) If $a_{12}^{-} b_{2}^{+}-a_{12}^{+} b_{2}^{-}<0$ and $a_{12}^{+} a_{22}^{-}-a_{12}^{-} a_{22}^{+} \geq 0$, then it has two families of closed sliding poly-trajectories and a hyperbolic closed sliding poly-trajectory. 

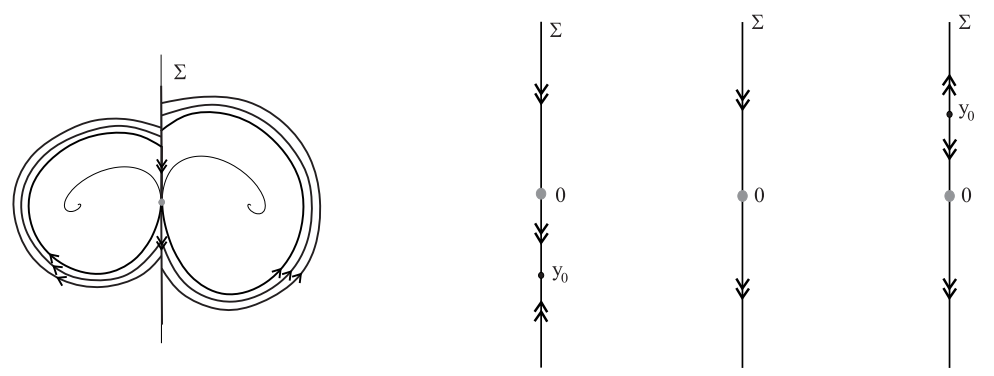

Figure 19: Possible closed sliding poly-trajectories for a PWL vector field with configuration given on line 10 of Table 3 and flows on $\Sigma$. The second and third figure correspond to item (a) and the last corresponds to item (b).

(c) If $a_{12}^{-} b_{2}^{+}-a_{12}^{+} b_{2}^{-}=0$ and $a_{12}^{+} a_{22}^{-}-a_{12}^{-} a_{22}^{+}>0$, then it has only a hyperbolic closed sliding poly-trajectory.

(d) If $a_{12}^{-} b_{2}^{+}-a_{12}^{+} b_{2}^{-}>0$, or $a_{12}^{-} b_{2}^{+}-a_{12}^{+} b_{2}^{-}=0$ and $a_{12}^{+} a_{22}^{-}-a_{12}^{-} a_{22}^{+}<0$, then it does not have closed sliding poly-trajectories.

The flows on $\Sigma$ are given in Figure 17.

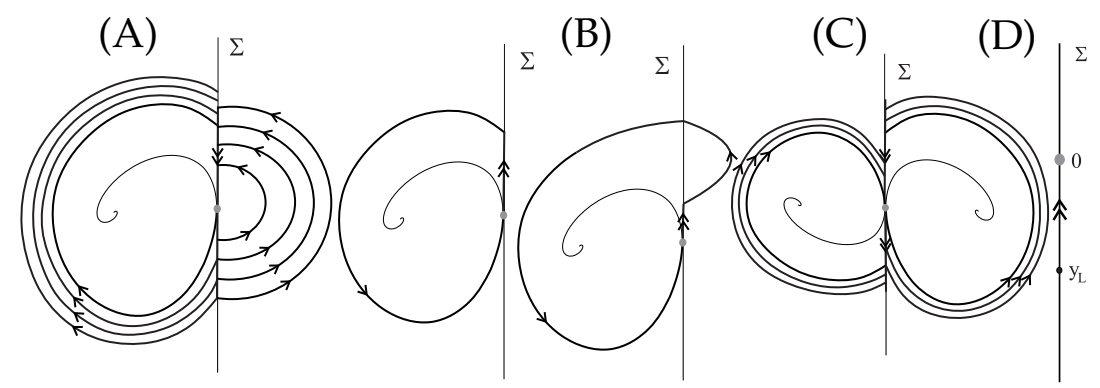

Figure 20: The figures (A), (B) and (C) represent the possible closed sliding polytrajectories for a PWL vector field with configuration given on lines 12, 20 and 23 of Table 3. The figure (D) represents the flow on $\Sigma$ for a PWL vector field with configuration given on line 20 of Table 3 .

Proposition 15. Consider a PWL vector field with configuration given on line 17 of Table 3. Let $a=a_{12}^{+} a_{22}^{-}-a_{12}^{-} a_{22}^{+}$and $b=a_{12}^{+} b_{2}^{-}-a_{12}^{-} b_{2}^{+}-a_{22}^{+} b_{1}^{-}$.

(a) If $a>0$, or $a \leq 0$ and $b<0$, or $a<0$ and $b>0$, then it can have or not $a$ hyperbolic closed sliding poly-trajectory.

(b) If $a=0$ and $b \geq 0$ or, $a<0$ and $b=0$, then it does not have closed sliding poly-trajectories.

Proposition 16. Consider a PWL vector field with configuration given on line 20 of Table 3. Let $a=a_{12}^{+} a_{22}^{-}-a_{12}^{-} a_{22}^{+}$and $b=a_{12}^{+} b_{2}^{-}-a_{12}^{-} b_{2}^{+}-a_{22}^{+} b_{1}^{-}$. If $a \leq 0$ or $b \leq 0$, then it can have a hyperbolic closed sliding poly-trajectory. 


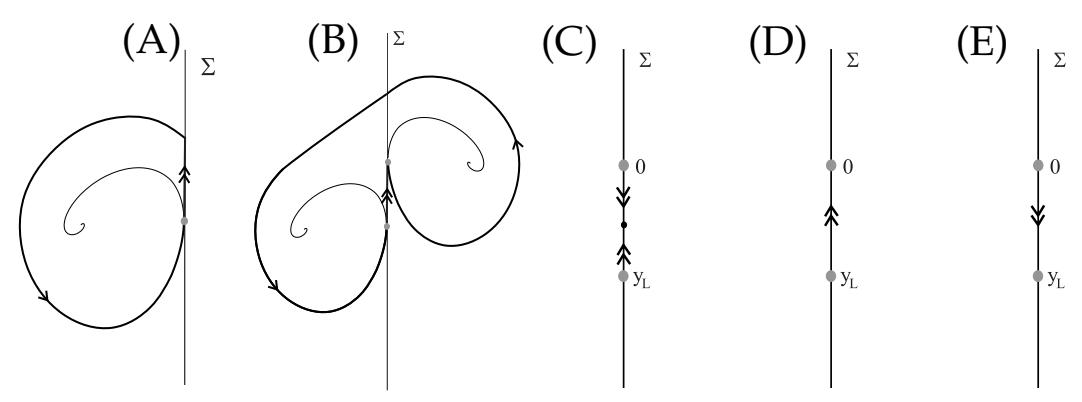

Figure 21: The figures (A) and (B) represent the possible closed sliding poly-trajectories for a PWL vector field with configuration given on line 17 of Table 3. The figures (C), (D) and (E) represent the flows on $\Sigma$ for a PWL vector field corresponding to this line.

Proposition 17. Consider a PWL vector field with configuration given on line 23 of Table 3.

(a) If $a_{12}^{+} a_{22}^{-}-a_{12}^{-} a_{22}^{+}>0$ or $a_{12}^{+} a_{22}^{-}-a_{12}^{-} a_{22}^{+}<0$, then it has at lest one family of closed sliding poly-trajectories and one hyperbolic closed sliding poly-trajectory.

(b) If $a_{12}^{+} a_{22}^{-}-a_{12}^{-} a_{22}^{+}=0$, then it has two families of closed sliding poly-trajectories and two hyperbolic closed sliding poly-trajectories.

The flows on $\Sigma$ are given on Figure 16 (A), (B) and (C). The Figures (A) and (B) corresponding to item (a) and the Figure (B) corresponding to item (b).

Proposition 18. Consider a PWL vector field with configuration given on line 24 of Table 3. Let $a=a_{12}^{+} a_{22}^{-}-a_{12}^{-} a_{22}^{+}$.

(a) If $a>0$, then it has at lest one hyperbolic closed sliding poly-trajectory.

(b) If $a<0$, then it can have or not one or two hyperbolic closed sliding poly-trajectories.

(c) If $a=0$, then it has two hyperbolic closed sliding poly-trajectories.

Proposition 19. Consider a PWL vector field with configuration given on line 25 of Table 3.

(a) If $a_{12}^{-} b_{2}^{+}-a_{12}^{+} b_{2}^{-}<0$ and $a_{12}^{+} a_{22}^{-}-a_{12}^{-} a_{22}^{+} \leq 0$, then it has two families of closed sliding poly-trajectories and a hyperbolic closed sliding poly-trajectory.

(b) If $a_{12}^{-} b_{2}^{+}-a_{12}^{+} b_{2}^{-}<0$ and $a_{12}^{+} a_{22}^{-}-a_{12}^{-} a_{22}^{+}>0$, then it has a family of closed sliding poly-trajectories and it can have more one family of closed sliding poly-trajectories and a hyperbolic closed sliding poly-trajectory.

(c) If $a_{12}^{-} b_{2}^{+}-a_{12}^{+} b_{2}^{-}=0$ and $a_{12}^{+} a_{22}^{-}-a_{12}^{-} a_{22}^{+}<0$, then it has only a hyperbolic closed sliding poly-trajectory.

(d) If $a_{12}^{-} b_{2}^{+}-a_{12}^{+} b_{2}^{-}>0$ or, $a_{12}^{-} b_{2}^{+}-a_{12}^{+} b_{2}^{-}=0$ and $a_{12}^{+} a_{22}^{-}-a_{12}^{-} a_{22}^{+}>0$, then it does not have closed sliding poly-trajectories. 


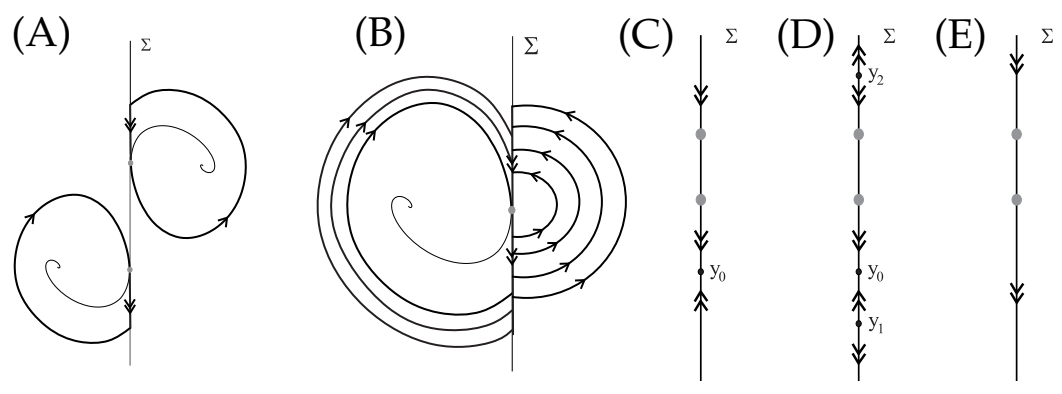

Figure 22: The figures (A) and (B) represent the possible closed sliding poly-trajectories for a PWL vector field with configuration given on lines 24 and 25 of Table 3, respectively. The figures (C), (D) and (E) represent the flows on $\Sigma$ for a PWL vector field with configuration given on line 24 of Table 3 , for $a>0, a<0$ and $a=0$, respectively.

The flows on $\Sigma$ are given in Figure 17. The first and third figure correspond to item (a), the second figure corresponds to item (b), the seventh figure corresponds to item (c) and the remaining figures correspond to item (d).

Proposition 20. Consider a PWL vector field with configuration given on line 26 of Table 3. Let $a=a_{12}^{+} a_{22}^{-}-a_{12}^{-} a_{22}^{+}$and $b=a_{12}^{+} b_{2}^{-}-a_{12}^{-} b_{2}^{+}-a_{22}^{+} b_{1}^{-}$.

(a) If $a>0$, or $a<0$ and $b>0$, or $a=0$ and $b<0$ then it can have or not $a$ hyperbolic closed sliding poly-trajectory.

(b) If $a<0$ and $b \leq 0$, or $a=0$ and $b>0$ then it has a hyperbolic closed sliding poly-trajectory.
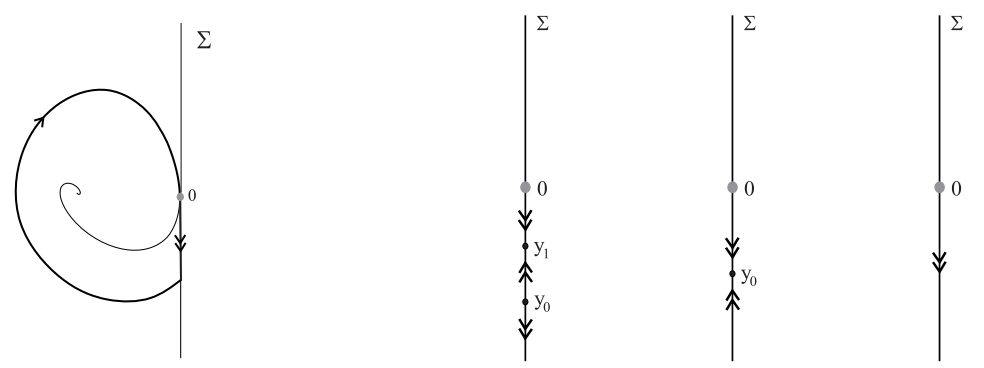

Figure 23: Possible closed sliding poly-trajectory for a PWL vector field with configuration given on line 26 of Table 3 and flows on $\Sigma$. The second and third figure correspond to item (a) and the last figure corresponds to item (b).

Proof of Theorem 3. The proof of Theorem 3 is immediate consequence of Propositions $1-20$.

Now, we present two examples which the PWL vector fields have closed sliding poly-trajectories. 
Example 1. Consider the PWL vector field given by

$$
F(x, y)=\left\{\begin{array}{lll}
(-y+1, x+y-2) & \text { if } \quad x \geq 0 \\
(y+1,-x-y-2) & \text { if } \quad x \leq 0
\end{array}\right.
$$

It corresponds to configuration given on line 24 of Table 3 with $\Sigma^{s}=\{(0, y)$ : $y>1\}, \Sigma^{c}=\{(0, y):-1<y<1\}$ and $\Sigma^{e}=\{(0, y): y<-1\}$. We have two fold points $(0,-1),(0,1)$ and $a_{12}^{+}=-1, a_{12}^{-}=1, a_{22}^{+}=1$ and $a_{22}^{-}=-1$. So, $a=a_{12}^{+} a_{22}^{-}-a_{12}^{-} a_{22}^{+}=0$. By Proposition 18, there is two closed sliding polytrajectories. In fact, the sliding vector field is given by $F^{\Sigma}(y)=(0,-1)$. Thus, the flow on $\Sigma$ is given on Figure $22(\mathrm{E})$ and the orbit which has $\alpha$-limit $(1,0)$, has $\omega$-limit $(1,0)$, the orbit which has $\omega$-limit $(-1,0)$, has $\alpha$-limit $(-1,0)$, as presented on Figure $22((\mathrm{~B}))$.

Example 2. Consider the PWL vector field given by

$$
F(x, y)=\left\{\begin{array}{lll}
(-y, x+y+1) & \text { if } \quad x \geq 0 \\
(y,-x-y-2) & \text { if } \quad x \leq 0
\end{array}\right.
$$

It corresponds to configuration given on line 25 of Table 3 with $\Sigma^{s}=\{(0, y)$ : $y>0\}$ and $\Sigma^{e}=\{(0, y): y<0\}$. We have a fold point $(0,0)$ and $a_{12}^{+}=-1, a_{12}^{-}=$ $1, a_{22}^{+}=1, a_{22}^{-}=-1, b_{2}^{+}=1$ and $b_{2}^{-}=-2$. So, $a_{12}^{+} a_{22}^{-}-a_{12}^{-} a_{22}^{+}=0$ and $a_{12}^{-} b_{2}^{+}-$ $a_{12}^{+} b_{2}^{-}=-1<0$. By Proposition $19, F^{-}$has a closed sliding poly-trajectory and $F^{+}$has a family of closed sliding poly-trajectories. In fact, the sliding vector field is given by $F^{\Sigma}(y)=(0,-1 / 2)$. Thus, the flow on $\Sigma$ is given on Figure 17 (third) and the consequence is immediate.

\section{Known Examples of Crossing Poly-Trajectories}

Now, we present a list of known examples of crossing poly-trajectory for the saddle-saddle, saddle-focus and focus-focus cases. This examples were obtained recently.

Example 3 (J. C. Arts, J. Llibre, J. C. Medrado and M. A. Teixeira, 2011). In [3] the authors proved that vector field (1) with

$$
\begin{aligned}
& F^{+}(x, y)=\left(\begin{array}{cc}
a^{+} & 1+b^{+} \\
1+c^{+} & d^{+}
\end{array}\right)\left(\begin{array}{c}
x-1-\alpha^{+} \\
y-\beta^{+}
\end{array}\right), \\
& F^{-}(x, y)=\left(\begin{array}{cc}
a^{-} & 1+b^{-} \\
1+c^{-} & d^{-}
\end{array}\right)\left(\begin{array}{c}
x+1-\alpha^{-} \\
y-\beta^{-}
\end{array}\right)
\end{aligned}
$$

satisfying $0 \leq\left|a^{ \pm}\right|,\left|b^{ \pm}\right|,\left|c^{ \pm}\right|,\left|d^{ \pm}\right|,\left|\alpha^{ \pm}\right|,\left|\beta^{ \pm}\right| \ll 1$ and with two real saddles, has at most 2 hyperbolic crossing poly-trajectories.

Example 4 (J. Llibre, M. A. Teixeira and J. Torregrossa, 2012). In [5] the authors proved that vector field (1) with

$$
\begin{aligned}
& F^{+}(x, y)=\left(\frac{1-\delta}{\pi} \log \left(\frac{11(5-3 \varepsilon)}{14(3 \varepsilon+5)}\right) x+y,-x+\frac{1-\delta}{\pi} \log \left(\frac{11(5-3 \varepsilon)}{14(3 \varepsilon+5)}\right) y\right), \\
& F^{-}(x, y)=\left(\frac{3}{2}(1+\varepsilon) x+y-\frac{3}{10}, 4 x-\frac{3}{2}(1-\varepsilon) y+\frac{67}{10}\right)
\end{aligned}
$$


satisfying $0<\delta \ll \varepsilon \ll 1$, is of kind saddle-focus and has 2 hyperbolic crossing poly-trajectories.

Example 5 (J. Llibre and E. Ponce, 2011). In [4] the authors proved that the system

$$
\begin{cases}A^{+} \mathbf{X}, & \text { if } \quad x \geq 1 \\ A^{-} \mathbf{X}, & \text { if } \quad x<1\end{cases}
$$

where $\mathbf{X}=(x, y)^{T}$ with

$$
A^{+}=\left(\begin{array}{cc}
\frac{19}{500} & -\frac{1}{10} \\
\frac{1}{10} & \frac{19}{500}
\end{array}\right) \text {, and } A^{-}=\left(\begin{array}{cc}
1 & -5 \\
\frac{377}{1000} & -\frac{13}{10}
\end{array}\right)
$$

has 3 crossing poly-trajectories.

The configurations of the PWL vector fields of the previous examples are given in Propositions 21, 22 and 23.

Proposition 21. The configuration of Example 3 is given on line 11 (by using item (i) of relation $R_{1}$ ) or 12 (by using $f_{1}(x, y)=\left(x,-y\right.$ ) in item (ii) of relation $R_{1}$ ) of Table 1 .

Proof. Using the change of coordinates as shown in the paper [3], we have the following vector field

$$
\begin{aligned}
& F^{+}(x, y)=\left(\begin{array}{ll}
0 & 1 \\
1 & d_{1}
\end{array}\right)\left(\begin{array}{c}
x-1-\alpha \\
y
\end{array}\right) \\
& F^{-}(x, y)=\left(\begin{array}{cc}
0 & 1 \\
1 & d_{2}
\end{array}\right)\left(\begin{array}{c}
x+1 \\
y
\end{array}\right) .
\end{aligned}
$$

It has a crossing poly-trajectory and 2 real saddles. Moreover the manifolds determined by the eigenvalues of the linear part of $F^{-}, F^{+}$are not parallel to $\Sigma$. We have $F_{1}^{-}(x, y)=F_{1}^{+}(x, y)=y$, so there is a fold point $F_{L}=F_{R}=(0,0)$. A simple computer gives $\Sigma^{c}=\{(0, y): y \in \mathbb{R} \backslash\{0\}\}$. So, the parameters $n_{1}$ and $n_{2}$ are given by $\mathbf{C C}$ and $\mathbf{R} \mathbf{R}$ respectively. Because $F^{-}(0, y), F(0, y)>0$ for $y>0$ and $F^{-}(0, y), F(0, y)<0$ for $y<0, n_{3}, n_{4}, n_{5}$ and $n_{6}$ are given respectively by $\mathbf{W}_{L}^{u} \cap \Sigma>\mathbf{F}_{L}, \mathbf{W}_{L}^{s} \cap \Sigma<\mathbf{F}_{L}, \mathbf{W}_{L}^{u} \cap \Sigma<\mathbf{F}_{R}$ and $\mathbf{W}_{L}^{s} \cap \Sigma>\mathbf{F}_{R}$.

The second hyperbolic crossing poly-trajectory is obtained adding $\left(\varepsilon^{3}, 0\right)$ to $F^{+}$and subtracting to $F^{-}$, with $\varepsilon>0$ sufficiently small. The calculations are analogous and we obtain the same parameters $n_{2}, n_{3}, n_{4}, n_{4}$ and $n_{6}$. Now we have a escaping region given by,

$$
\Sigma^{e}=\left\{(0, y):-\varepsilon^{3}<y<\varepsilon^{3}\right\} .
$$

Then, the parameter $n_{1}$ is given by CEC. 
Proposition 22. The configuration of Example 4 is given on line 13 of Table 2 (by using item (i) of relation $R_{2}$ ).

Proof. As the authors presented in [5], for $0<\delta \ll \varepsilon \ll 1$ the vector field (1) with $F^{+}$and $F^{-}$given in Example 4 has a virtual stable focus at $(0,0)$, with eigenvalues given by

$$
(1-\delta) \log \left(\frac{11(5-3 \varepsilon)}{14(3 \varepsilon+5)}\right) \pm i
$$

and has a real saddle at the equilibrium point

$$
\left(\frac{9 \varepsilon+125}{5\left(9 \varepsilon^{2}-25\right)},-\frac{3(67 \varepsilon+75)}{5\left(9 \varepsilon^{2}-25\right)}\right)
$$

with eigenvalues $(3 \varepsilon \pm 5) / 2$. The manifolds are given by the straight lines $y=x+14 /(5-3 \varepsilon)$ and $y=-4 x-11 /(5+3 \varepsilon)$. We have $F_{1}^{-}(0, y)=y-3 / 10$ and $F_{1}^{+}(0, y)=y$ and thus $\Sigma^{c}=\{(0, y): y>3 / 10$ or $y<0\}, \Sigma^{e}=\{(0, y)$ : $0<y<3 / 10\}$. Thus, the parameters $m_{1}, m_{2}, m_{3}, m_{4}, m_{5}$ and $m_{6}$ are given by $\mathbf{C C}$, $\mathbf{R V}, \mathbf{W}_{L}^{u} \cap \Sigma>\mathbf{F}_{L}, \mathbf{W}_{L}^{s} \cap \Sigma<\mathbf{F}_{L},+1$ and $<0$, respectively.

Proposition 23. The configuration of Example 5 is given on line 20 of Table 3.

Proof. We have that $F_{1}^{-}(1, y)=1-5 y$ and $F_{1}^{+}(1, y)=19 / 500-1 / 10 y$ and thus $\Sigma^{c}=\{(1, y): y<1 / 5$ or $y>19 / 50\}, \Sigma^{e}=\{(1, y): 1 / 5<y<19 / 50\}$. Clearly $(0,0)$ is a virtual unstable focus for the system $\mathbf{X}=A^{+} \mathbf{X}$ and a real stable focus for the system $\mathbf{X}=A^{-} \mathbf{X}$. In fact, the eigenvalues of $A^{-}$and $A^{+}$are given by

$$
\frac{19}{500} \pm \frac{1}{10} i, \quad-\frac{3}{20} \pm \frac{3}{4} i
$$

So, the parameters $s_{1}, s_{2}, s_{3}, s_{4}, s_{5}$ and $s_{6}$ are given respectively by CEC, RV, $-1,<0,-1,>0$.

\section{References}

[1] C. A. Buzzi, T. de Carvalho and P. R. da Silva Closed poly-trajectories and Poincar index of non-smooth vector fields on the plane, Journal of Dynamical and Control Systems., 19, (2013).

[2] E. Freire, E. Ponce, F. Torres Canonical discontinuous planar piecewise linear systems, SIAM J. Appl. Dyn. Syst., 11, 181-211 (2012).

[3] J. C. Arts, J. Llibre, J. C. Medrado and M. A. Teixeira Piecewise linear differential systems with two real saddles, Prepublications del Dept. de Matemtiques de la UAB, 37, (2011).

[4] J. Llibre and E. Ponce Three nested limit cycles in discontinuous piecewise linear differential systems with two zones, Dyn. Contin. Discrete Impuls. Syst. Ser. B Appl. Algorithms, 3, (2012). 
[5] J. Llibre, M. A. Teixeira and J. Torregrossa, On the maximum number of limit cycles of discontinuous piecewise Linear differential systems with a straight line of separation, Preprint.

[6] J. Llibre, P. R. da Silva and M. A. Teixeira, Sliding Vector Fields via Slow-Fast Systems Bulletin of the Belgian Mathematical Society Simon Stevin 15 (2008), 851-869.

[7] J. Sotomayor and A. L. F. Machado, Structurally stable discontinuous vector fields in the plane, Qualitative Theory of Dynamical Sistems 3, 227-250 (2002).

[8] M. di Bernardo, C. J. Budd, A. R. Champneys and P. Kowalczyk Piecewisesmooth dynamical systems Appl. Math. Sci. Series vol 163 (London: Springer)

[9] S. Huan and X. Yang, On the number of limit cycles in general planar piecewise linear systems, Discrete Contin. Dynam Systems, (2012).

[10] T. de Carvalho and D. J. Tonon, Generic Bifurcations of Planar Filippov Systems via Geometric Singular Perturbations, Bulletin of the Belgian Mathematical Society Simon Stevin, 18, (2011), 861-881.

Departamento de Matemática - IBILCE-UNESP,

Rua C. Colombo, 2265, CEP 15054-000 S. J. Rio Preto,

São Paulo, Brazil.

email:jrezmoraes@gmail.com, prs@ibilce.unesp.br 